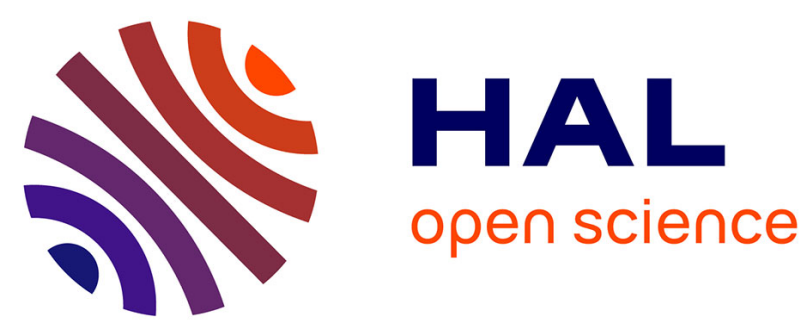

\title{
Asymptotic estimates of the convergence of classical Schwarz waveform relaxation domain decomposition methods for two-dimensional stationary quantum waves
} Xavier Antoine, Fengji Hou, Emmanuel Lorin

\section{- To cite this version:}

Xavier Antoine, Fengji Hou, Emmanuel Lorin. Asymptotic estimates of the convergence of classical Schwarz waveform relaxation domain decomposition methods for two-dimensional stationary quantum waves. ESAIM: Mathematical Modelling and Numerical Analysis, 2018, 52 (4), pp.1569-1596. $10.1051 / \mathrm{m} 2 \mathrm{an} / 2017048$. hal-01431866

\author{
HAL Id: hal-01431866 \\ https://hal.science/hal-01431866
}

Submitted on 11 Jan 2017

HAL is a multi-disciplinary open access archive for the deposit and dissemination of scientific research documents, whether they are published or not. The documents may come from teaching and research institutions in France or abroad, or from public or private research centers.
L'archive ouverte pluridisciplinaire $\mathbf{H A L}$, est destinée au dépôt et à la diffusion de documents scientifiques de niveau recherche, publiés ou non, émanant des établissements d'enseignement et de recherche français ou étrangers, des laboratoires publics ou privés. 


\title{
Asymptotic estimates of the convergence of classical Schwarz waveform relaxation domain decomposition methods for two-dimensional stationary quantum waves
}

\author{
Xavier Antoine ${ }^{\mathrm{a}}$, Fengji Hou ${ }^{\mathrm{b}}$, Emmanuel Lorin ${ }^{\mathrm{b}, \mathrm{c}}$ \\ ${ }^{a}$ Institut Elie Cartan de Lorraine, Université de Lorraine, Inria Nancy-Grand Est, F-54506 Vandoeuvre-lès-Nancy Cedex, \\ France \\ ${ }^{b}$ School of Mathematics and Statistics, Carleton University, Ottawa, Canada, K1S 5B6 \\ ${ }^{c}$ Centre de Recherches Mathématiques, Université de Montréal, Montréal, Canada, H3T1J4
}

\begin{abstract}
This paper is devoted to the analysis of convergence of Schwarz Waveform Relaxation (SWR) domain decomposition methods (DDM) for solving the stationary linear and nonlinear Schrödinger equations by the imaginary-time method. Although SWR are extensively used for numerically solving high-dimensional quantum and classical wave equations, the analysis of convergence and of the rate of convergence is still largely open for variable coefficients linear and nonlinear equations. The aim of this paper is to tackle this problem for both the linear and nonlinear Schrödinger equations in the two-dimensional setting. By extending ideas and concepts presented earlier [12] and by using pseudodifferential calculus, we prove the convergence and determine some approximate rates of convergence of the two-dimensional Classical SWR method for two subdomains with smooth boundary. Some numerical experiments are also proposed to validate the analysis.
\end{abstract}

Keywords: Schwarz Waveform Relaxation. Domain decomposition method. Convergence rate. Schrödinger equation. Gross-Pitaevskii equation. Absorbing boundary conditions. Pseudodifferential operator theory. Symbolical asymptotic expansion. Stationary states. Imaginary-time. Continuous Normalized GradientFlow.

\section{Introduction}

Let us consider the following initial boundary-value problem: find the complex-valued wavefunction $u(\mathbf{x}, t)$ solution to the real-time cubic nonlinear Schrödinger equation on $\mathbb{R}^{d}, d \geqslant 1$,

$$
\left\{\begin{array}{l}
i \partial_{t} u=-\triangle u+V(\mathbf{x}) u+\nu|u|^{2} u, \mathbf{x} \in \mathbb{R}^{d}, t>0 \\
u(\mathbf{x}, 0)=u_{0}(\mathbf{x}), \mathbf{x} \in \mathbb{R}^{d}
\end{array}\right.
$$

with initial condition $u_{0}$. The real-valued space-dependent smooth potential $V$ is positive (respectively negative) for attractive (respectively repulsive) interactions. The nonlinearity strength $\nu$ is a real-valued constant which is positive (respectively negative) for a focusing (respectively defocusing) nonlinearity. If $\nu=0$, then we will speak about the time-dependent Linear Schrödinger Equation (LSE). In the Physics literature, the first equation of system (1) is also called the Gross-Pitaevskii Equation (GPE) [2, 11, 16], when considering Bose-Einstein Condensates (BEC) (see e.g. [38, 39]). The computation of stationary states, e.g. ground state and excited states, is a major question in quantum physics, most particularly for BECs. Such a problem corresponds $[9,10,11,16,17,18,19]$ to computing a real number $\mu$ and a space dependent function $\phi$ which satisfies the equation

$$
\mu \phi(\mathbf{x})=-\triangle \phi(\mathbf{x})+V(\mathbf{x}) \phi(\mathbf{x})+\nu|\phi(\mathbf{x})|^{2}, \mathbf{x} \in \mathbb{R}^{d},
$$

Email addresses: xavier.antoine@univ-lorraine.fr (Xavier Antoine), fengji.hou@gmail.com (Fengji Hou), elorin@math.carleton.ca (Emmanuel Lorin) 
under the normalization constraint

$$
\|\phi\|_{L^{2}\left(\mathbb{R}^{d}\right)}^{2}:=\int_{\mathbb{R}^{d}}|\phi(\mathbf{x})|^{2} d \mathbf{x}=1 .
$$

If we define the total energy of the system as

$$
E_{\nu}(\chi):=\int_{\mathbb{R}^{d}}|\nabla \chi(\mathbf{x})|^{2}+V(\mathbf{x})|\chi(\mathbf{x})|^{2}+\frac{\nu}{2}|\chi(\mathbf{x})|^{4} d \mathbf{x},
$$

then a stationary state is such that

$$
E_{\nu}(\phi):=\min _{\|\chi\|_{L^{2}\left(\mathbb{R}^{d}\right)}=1} E_{\nu}(\chi) .
$$

Once it is obtained, the eigenvalue $\mu$ (also called chemical potential) can be computed through the eigenfunction $\phi$ by using the expression

$$
\mu:=\mu_{\nu}(\phi)=E_{\nu}(\phi)+\frac{\nu}{2} \int_{\mathbb{R}^{d}}|\phi(\mathbf{x})|^{4} d \mathbf{x} .
$$

Existence and uniqueness results for the minimizers corresponding to a ground state (global minimizer) or excited states (local minimizers) can be found in the literature [16]. More general versions of the GPE include rotational terms, complex nonlinear (nonlocal) functions and coupled species of cold atomic gases $[2,11,15,16,40]$.

To numerically determine $(\mu, \phi)$, a well-known method is the so-called imaginary time method $[9,10,11$, $16,17,18,19,20,23]$ which is also designated as a Continuous Normalized Gradient Flow (CNGF) method in the Applied Mathematics literature. It consists in solving (1) in imaginary-time, i.e. setting $t \rightarrow i t$. This transformation leads to the formulation

$$
\left\{\begin{array}{l}
\partial_{t} \phi(\mathbf{x}, t)=-\nabla_{\phi^{*}} E_{\nu}(\phi) \\
=\triangle \phi(\mathbf{x}, t)-V(\mathbf{x}) \phi(\mathbf{x}, t)-\nu|\phi|^{2} \phi(\mathbf{x}, t), \mathbf{x} \in \mathbb{R}^{d}, t_{n}<t<t_{n+1}, \\
\phi\left(\mathbf{x}, t_{n+1}\right):=\phi\left(\mathbf{x}, t_{n+1}^{+}\right)=\frac{\phi\left(\mathbf{x}, t_{n+1}^{-}\right)}{\left\|\phi\left(\cdot, t_{n+1}^{-}\right)\right\|_{L^{2}\left(\mathbb{R}^{d}\right)}}, \\
\phi(\mathbf{x}, t)=\phi_{0}(\mathbf{x}), \mathbf{x} \in \mathbb{R}^{d}, \text { with }\left\|\phi_{0}\right\|_{L^{2}\left(\mathbb{R}^{d}\right)}=1 .
\end{array}\right.
$$

In the above system of equations, $t_{0}:=0<t_{1}<\ldots<t_{n+1}<\ldots$ are discrete times, $\phi_{0}$ is an initial data for the time marching algorithm discretizing the projected gradient method and $\lim _{t \rightarrow t_{n}^{ \pm}} \phi(\mathbf{x}, t)=\phi\left(\mathbf{x}, t_{n}^{ \pm}\right)$. It can be proven in the one-dimensional case [18] that the energy is diminishing for positive $V$ and $\nu=0$.

In this paper, we study the convergence of Schwarz Waveform Relaxation Domain Decomposition Methods (DDM) for solving the stationary two-dimensional linear Schrödinger equation and Gross-Pitaevskii equation using the imaginary-time method. Thanks to pseudodifferential calculus, we study the SWR-DDM for the Schrödinger equation with variable potentials and with non-flat subdomain interfaces. This paper is the sequel of [12] where the one-dimensional algorithm was analyzed in details. Domain decomposition methods are particularly well-adapted for the parallel solution of linear systems that appear in finite difference and finite element methods. Among the various domain decomposition methods [24, 28], we focus our attention here on the Classical Schwarz Waveform Relaxation (CSWR) DDM [1, 13, 25, 26, 27, 28, 29, 30, 31, 32, 36]. Even if this method has received much attention over the past years for many applications, to the best of the authors' knowledge, the first application to the Schrödinger equation can be found in [32]. The authors consider the real-time linear one-dimensional Schrödinger equation with a constant potential. Well-posedness results are stated, and continuous and discrete analysis of the algorithm are developed. Another recent paper for the Schrödinger equation is [14], where the algorithms are analyzed for a one-dimensional time-dependent linear Schrödinger equation that includes ionization and recombination by intense electric field. In [22], the authors study the numerical performance of Schwarz waveform relaxation methods for the one-dimensional dynamical solution of the LSE with a general potential, most particularly regarding their efficiency when a GPU implementation is considered. More recently the same authors have numerically studied [21] the CSWR 
and OSWR algorithms for the two-dimensional Schrödinger equation. The behavior of the method shows that it can lead to fast and robust algorithms for complex linear problems. In [35], domain decomposition methods have been developed when using geometric optics and frozen gaussian approximations for computing the solution to linear Schrödinger equations under and beyond the semi-classical regime.

To conclude this overview, we recall the general principle of the Schwarz Waveform Relaxation algorithm, applied to two two-dimensional subdomains. We first introduce two open sets $\Omega_{\varepsilon}^{ \pm}$, with boundary $\Gamma_{\varepsilon}^{ \pm}:=\partial \Omega_{\varepsilon}^{ \pm}$, such that $\mathbb{R}^{2}=\Omega_{\varepsilon}^{+} \cup \Omega_{\varepsilon}^{-}$with overlapping region $\Omega_{\varepsilon}^{+} \cap \Omega_{\varepsilon}^{-}$, where $\varepsilon$ is a non-negative parameter. We denote by $\psi^{ \pm}$the solution to the LSE/GPE in $\Omega_{\varepsilon}^{ \pm}$. Solving the GPE by Schwarz waveform domain decomposition (see [14] for instance) requires some transmission conditions at the subdomain interfaces. More specifically, for any Schwarz iteration $k \geqslant 1$, the equation in $\Omega_{\varepsilon}^{ \pm}$reads, for a given $T>0$

$$
\left\{\begin{array}{l}
P \cdot \psi^{ \pm,(k)}=0, \text { on } \Omega_{\varepsilon}^{ \pm} \times(0, T), \\
\mathcal{B}_{ \pm} \psi^{ \pm,(k)}=\mathcal{B}_{ \pm} \psi^{\mp,(k-1)}, \text { on } \Gamma_{\varepsilon}^{ \pm} \times(0, T), \\
\psi^{ \pm,(k)}(\cdot, 0)=\psi_{0}(\cdot) \text { on } \Omega_{\varepsilon}^{ \pm} .
\end{array}\right.
$$

The notation $\psi^{ \pm,(k)}$ stands for the solution $\psi^{ \pm}$in $\Omega_{\varepsilon}^{ \pm} \times(0, T)$ at Schwarz iteration $k \geqslant 0$. Initially $\psi^{ \pm,(0)}$ are two given functions defined in $\Omega_{\varepsilon}^{ \pm}$, typically taken null if no further information is provided. The operator $\mathcal{B}_{ \pm}$characterizes the type of SWR algorithm. In the CSWR case, $\mathcal{B}_{ \pm}$is simply the identity operator, $\mathcal{B}_{ \pm}=\partial_{\mathbf{n}^{ \pm}}+\gamma \mathrm{Id}\left(\gamma \in \mathbb{R}_{+}^{*}\right)$ for Robin SWR, and $\mathcal{B}_{ \pm}$is a nonlocal Dirichlet-to-Neumann-like (DtN) pseudodifferential operator for Optimized SWR. We refer to $[14,32]$ for further reading.

The goal of the present paper is to contribute to the understanding of the behavior of multi-dimensional Schwarz waveform relaxation DDMs, in particular the effect of the interface curvature on the rate convergence. In Section 2, we recall important pseudodifferential calculus definitions and results which will be useful for the analysis of convergence of the two-dimensional SWR algorithm. Then, we derive in Section 3 some analytical estimates of the convergence rate for the CSWR two-domains decomposition method for the linear Schrödinger (with variable potential) and the Gross-Pitaevskii equations by using the CNGF method (3) in 2-d. To this aim, we propose an extension of the techniques developed e.g. in $[12,25,32]$ to variable coefficient equations. In particular, we make an intensive use of the theory of fractional pseudodifferential operators [33] and asymptotic symbolical calculus (see e.g. $[4,5,6,8]$ for some applications). Section 4 is devoted to numerical experiments validating and illustrating the analysis presented in this paper. Finally, we conclude in Section 5.

\section{Background on pseudodifferential operator calculus}

This section is devoted to the presentation of analytical and geometrical tools for constructing and analyzing Schwarz waveform relaxation domain decomposition methods in 2-d. The use of pseudodifferential calculus, will allow us, not only to analyze the SWR-DDM with non-flat interfaces, but will also be crucial to perform a Nirenberg factorization of the Schrödinger equation with non-constant coefficients, at the subdomain interfaces.

\subsection{Local parameterization}

Let $\Omega$ be a convex domain with smooth boundary $\Sigma$, and (positive) local curvature $\kappa=\kappa(s)$, at curvilinear abscissa $s$ which is then positive. We do not detail here all the calculations and refer to [6] for more explanations concerning the change of variables. For a point $M$ of $\Sigma$ with coordinates $(x, y)$, we designate by $\boldsymbol{\tau}$ the unitary tangential vector to $\Sigma$ at $M$, and $\mathbf{n}$ the outwardly directed unit normal vector. In the local coordinates system associated with $M$, a point $M^{\prime}$ in a local neighborhood of $M$ is connected to its coordinates $r$ and $s$. Since $\Omega$ is convex, the projection of the point $M^{\prime}$ onto the boundary $\Sigma$ is unique, giving hence its curvilinear abscissa $s$. The radial coordinates $r$ is the distance from point $M^{\prime}$ to its projection according to the outgoing unitary normal vector. Hence, $\Sigma$ can be denoted by $\Sigma_{0}$, if $\Sigma_{r}$ designates the 
parallel surface to $\Sigma$ at distance $r$. Since $\Sigma$ is convex, we can restrict ourselves to positive values of $r$, bounded from above by a small parameter $\varepsilon$, and so $r \in[0, \varepsilon]$. Now, the Laplacian in local coordinates $(r, s)$ writes down [6]

$$
\Delta_{r}=\partial_{r}^{2}+\kappa_{r} \partial_{r}+h^{-1} \partial_{s}\left(h^{-1} \partial_{s}\right)
$$

with the scaling factor $h: h=1+r \kappa$ and $\kappa_{r}$ the curvature at $M^{\prime}$ on the parallel surface $\Sigma_{r}: \kappa_{r}=h^{-1} \kappa$. For the sake of conciseness, we denote by $\tilde{u}$ the function $u$ written in the local system

$$
u(x, y, t)=\tilde{u}(r, s, t), \quad(x, y) \in \mathbb{R}^{2},(r, s) \in[0, \varepsilon] \times[a, b], t>0,
$$

and $V_{r}$ the locally rewritten potential function

$$
V(x, y, t)=V_{r}(r, s, t), \quad(x, y) \in \mathbb{R}^{2},(r, s) \in[0, \varepsilon] \times[a, b], t>0 .
$$

The Schrödinger equation for system (18) then becomes

$$
\left.\left.i \partial_{t} \tilde{u}+\partial_{r}^{2} \tilde{u}+\kappa_{r} \partial_{r} \tilde{u}+h^{-1} \partial_{s}\left(h^{-1} \partial_{s}\right) \tilde{u}+V_{r} \tilde{u}=0, \quad(r, s, t) \in[0, \varepsilon] \times[a, b] \times\right) 0, T\right],
$$

where $r$ and $s$ parameterize the domain $\Omega$ and $t>0$. In the sequel, we identify $u$ to $\tilde{u}$.

\subsection{Pseudodifferential operators for the two-dimensional case and associated symbolic calculus}

The functions that we consider in this chapter depend on the local spatial coordinates $r$ and $s$, and on time $t$. In this framework, the two-dimensional pseudodifferential operator calculus is realized through the partial Fourier transform $(s, t)$ of a function $f(r, s, t)$. We denote by $\xi$ (respectively $\tau$ ) the covariable of $s$ (respectively $t$ ). We have

$$
\mathcal{F}_{(t, s)}(f(r, s, t))(r, \xi, \tau)=\frac{1}{4 \pi^{2}} \int_{\mathbb{R}} \int_{\mathbb{R}} f(r, s, t) e^{-i t \tau} e^{-i s \xi} d t d s
$$

and we set $\mathcal{F}=\mathcal{F}_{(t, s)}$ in this section. A pseudodifferential operator $P\left(r, s, t, \partial_{s}, \partial_{t}\right)$ with symbol $p(r, s, t, \xi, \tau)$ is defined by

$$
P\left(r, s, t, \partial_{s}, \partial_{t}\right) u(r, s, t)=\mathcal{F}_{(t, s)}^{-1}(p(r, s, t, \xi, \tau) \widehat{u}(r, \xi, \tau))
$$

that is

$$
P\left(r, s, t, \partial_{s}, \partial_{t}\right) u(r, s, t)=\int_{\mathbb{R}} \int_{\mathbb{R}} p(r, s, t, \xi, \tau) \widehat{u}(r, \xi, \tau) e^{i t \tau} e^{i s \xi} d \tau d \xi
$$

where $\widehat{u}=\mathcal{F} u$.

The inhomogeneous pseudodifferential operator calculus that we use in the paper was introduced in [33] and applied e.g. in [3] to the construction of artificial boundary conditions. For the sake of conciseness, we only give the useful material needed here. Let $m$ be a real number and $\mathcal{O}$ an open subset of $\mathbb{R}^{2}$. Then (see [37]), the symbol class $S^{m}\left(\mathcal{O} \times \mathbb{R}^{+}\right)$denotes the linear space of $\mathcal{C}^{\infty}$ functions $a(r, s, t, \xi, \tau)$ in $\mathcal{O} \times \mathbb{R}^{+} \times \mathbb{R}^{2}$ such that for each $K \subseteq \mathcal{O}$ and for all integer indices $k, \alpha_{r}, \alpha_{s}, \ell$ and $\beta$, there exists a constant $C_{k, \alpha_{r}, \alpha_{s}, \ell, \beta}(K)$ such that

$$
\left|\partial_{t}^{k} \partial_{r}^{\alpha_{r}} \partial_{s}^{\alpha_{s}} \partial_{\tau}^{\ell} \partial_{\xi}^{\beta} a(r, s, t, \xi, \tau)\right| \leqslant C_{k, \alpha_{r}, \alpha_{s}, \ell, \beta}(K)\left(1+\tau^{2}+\xi^{4}\right)^{m-\beta-2},
$$

for all $(r, s) \in K, t \in \mathbb{R}^{+}$and $(\xi, \tau) \in \mathbb{R}^{2}$.

Let us set $E=(1,2)$. The smoothness of a pseudodifferential operator can be deduced from the homogeneity of its symbol with respect to $\left(\xi^{2}, \tau\right)$. Therefore, $\xi^{2}$ and $\tau$ are considered as homogeneous [3, 33]. This leads to the following definition.

Definition 2.1. A function $f(r, s, t, \xi, \tau)$ is said to be E-quasi homogeneous of order $m$ if and only if for all $\mu>0$ and for large $\left(\xi^{2}, \tau\right)$ we have

$$
f\left(r, s, t, \mu \xi, \mu^{2} \tau\right)=\mu^{m} f(r, s, t, \xi, \tau)
$$


The introduction of this last class of symbols is particularly well-adapted to studying heat-like and Schrödinger-type equations. For example, the operator with symbol $\lambda=\sqrt{-\tau-\xi^{2}}$ is first-order $E$-quasi homogeneous (with respect to $\left(\xi^{2}, \tau\right)$ ).

From now on, a $E$-quasi homogeneous pseudodifferential operator of order $m \in \mathbb{Z}$, denoted by $A \in \operatorname{OPS}_{E}^{m}$, is defined as an operator with a total symbol $a(r, s, t, \xi, \tau)$ admitting an asymptotic expansion in $E$-quasi homogeneous symbols

$$
a(r, s, t, \xi, \tau) \sim \sum_{j=0}^{+\infty} a_{m-j}(r, s, t, \xi, \tau),
$$

where the functions $a_{m-j}, j \in \mathbb{N}$, are $E$-quasi homogeneous of degree $m-j$. The meaning of $\sim$ in (13) is

$$
\forall \widetilde{m} \in \mathbb{N}, \quad a-\sum_{j=0}^{\widetilde{m}} p_{m-j} \in S_{E}^{m-(\widetilde{m}+1)} .
$$

A symbol $a$ satisfying the property (13) is denoted by $a \in S_{E}^{m}$ and the associated operators $A=O p(a)$ by $A \in \mathrm{OPS}_{E}^{m}$. Finally, we introduce $\mathrm{OPS}_{E}^{-\infty}$ as the intersection between all the classes $\mathrm{OPS}_{E}^{m}, m \in \mathbb{Z}$. For $P$ and $Q$ two pseudodifferential operators with respective symbols $p$ and $q$, and $m \in \mathbb{Z}$, we set

$$
P=Q \quad \bmod \mathrm{OPS}_{E}^{m}
$$

or equivalently

$$
p=q \quad \bmod S_{E}^{m}
$$

if the difference between the two symbols fulfills: $p-q \in S_{E}^{m}$. Finally, the composition formula for two operators $A$ and $B$ with respective symbols $\sigma(A)$ and $\sigma(B)$ writes

$$
\sigma(A B)=\sum_{|\alpha|=0}^{+\infty} \frac{(-i)^{|\alpha|}}{\alpha !} \partial_{(\xi, \tau)}^{\alpha} \sigma(A) \partial_{(t, s)}^{\alpha} \sigma(B) .
$$

Furthermore, if $\sigma(A) \in S_{E}^{m}$ and $\sigma(B) \in S_{E}^{n}$, then we have $\sigma(A B) \in S_{E}^{m+n}$. In (17), $\alpha$ is a multi-index $\left(\alpha_{1}, \alpha_{2}\right)$. We use the classical notations for multi-indices. In particular, its length $|\alpha|$ is defined by: $|\alpha|=\alpha_{1}+\alpha_{2}$. The factorial is defined by: $\alpha !=\alpha_{1} ! \alpha_{2}$ !, and we introduce the derivative according to $(\xi, \tau): \partial_{(\xi, \tau)}^{\alpha} \lambda=$ $\partial_{\xi}^{\alpha_{1}} \partial_{\tau}^{\alpha_{2}} \lambda(r, s, t, \xi, \tau)$. This class of operators allows to define an associated symbolic calculus [3, 33]. Finally, we have: $\sigma\left(\partial_{s}\right)=i \xi$ and $\sigma\left(\partial_{s}^{2}\right)=-\xi^{2}$.

\section{Asymptotic estimates of the contraction factor for the SWR algorithm}

This section is first devoted to the convergence of the Classical Schwarz Waveform Relaxation method applied to the LSE in imaginary-time

$$
\left\{\begin{array}{lll}
i \partial_{t} u+\triangle u-V(x, y) u & =0, & (x, y) \in \mathbb{R}^{2}, t>0 \\
u(x, y, 0) & =0, & (x, y) \in \mathbb{R}^{2},
\end{array}\right.
$$

with $u_{0} \in L^{2}\left(\mathbb{R}^{2}\right)$. We now introduce i) a fictitious domain $\Omega$ with smooth boundary $\Gamma$, and ii) a change of variables $x(r, s), y(r, s)$ parametrizing $\Gamma$, where $r$ and $s$ are respectively the radial coordinate and the curvilinear abscissa. We then rewrite (18) in generalized coordinates $(r, s)$, that is

$$
\left\{\begin{array}{l}
i \partial_{t} u+\partial_{r}^{2} u+\frac{1}{r} \partial_{r} u+\frac{1}{r^{2}} \partial_{s}^{2} u-V_{r}(r, s) u=0, \quad(r, s) \in \mathbb{R}_{+} \times \mathbb{R}_{+}, t>0, \\
u(r, s, 0)=u_{0}(x(r, s), y(r, s)), \quad(r, s) \in \mathbb{R}_{+} \times[0, \ell(\Omega)] .
\end{array}\right.
$$

We denote by $P_{r}$ the Schrödinger operator written in $(r, s)$-coordinates. Notice that the SWR method reads the same as (4), by replacing $P$ by $P_{r}$. This will also be explicitly stated in the following sections. Following 
a similar approach as in the one-dimensional case [12], we will first factorize the Schrödinger operator in term of outgoing and incoming wave operators at the subdomains interfaces. We limit the analysis to two domains with smooth boundary, and defined as follows: $\mathbf{0} \in \Omega_{\varepsilon}^{+}$and $\Omega_{\varepsilon}^{-} \cup \Omega_{\varepsilon}^{+}=\mathbb{R}^{2}$, and we assume that $\Gamma_{\varepsilon}^{+}:=\partial \Omega_{\varepsilon}^{+}$ and $\Gamma_{\varepsilon}^{-}:=\partial \Omega_{\varepsilon}^{-}$are parallel at distance $\varepsilon>0$, as represented in Fig. 1. The domain $\Omega_{\varepsilon}^{+}$can for instance
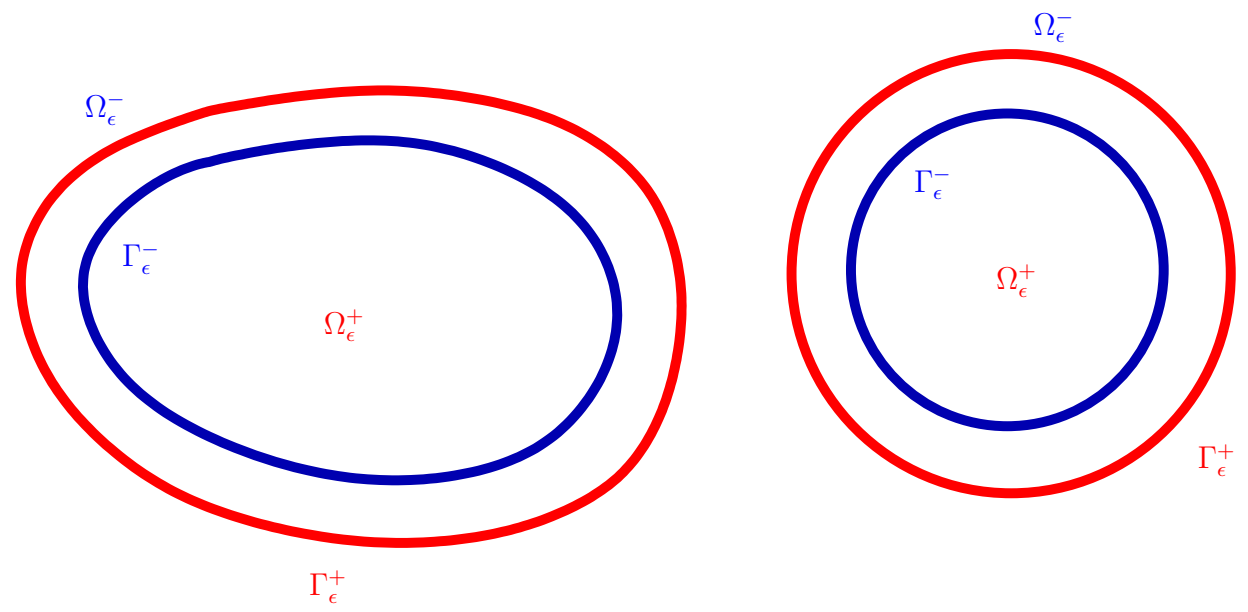

Figure 1: Two examples of admissible decomposition.

be chosen as a disc, $D\left(\mathbf{0}, R_{0}+\varepsilon / 2\right)$, of radius $R_{0}+\varepsilon / 2$ and center $\mathbf{0} \in \mathbb{R}^{2}$, and $\Omega_{\varepsilon}^{-}$as $\mathbb{R}^{2}-D\left(\mathbf{0}, R_{0}-\varepsilon / 2\right)$. This assumption allows for a simplification of the SWR algorithm and its mathematical analysis. Let us denote by $\kappa_{\varepsilon}^{ \pm}(s)$ the local curvature at $\Gamma_{\varepsilon}^{ \pm}$. Notice that $\kappa_{\varepsilon}^{+}$and $\kappa_{\varepsilon}^{-}$have opposite signs, and by construction $\kappa_{\varepsilon}^{+}>0$ and $\kappa_{\varepsilon}^{-}=-\left(1+\varepsilon \kappa_{\varepsilon}^{+}\right)^{-1} \kappa_{\varepsilon}^{+}<0$. As in [3], we introduce the scaling factor $h_{\varepsilon}^{ \pm}(r, s)=1 \mp r \kappa_{\varepsilon}^{ \pm}(s)$ and we denote by $\Gamma_{\varepsilon, r}^{ \pm}$, the parallel surface to $\Gamma_{\varepsilon}^{ \pm}$at distance $r \in[0, \varepsilon / 2]$. The curvature of $\Gamma_{\varepsilon, r}^{+}$is given by $\kappa_{\varepsilon, r}^{+}(r, s)=\left(h_{\varepsilon}^{+}(r, s)\right)^{-1} \kappa_{\varepsilon}^{+}(s)$. Similarly, $\kappa_{\varepsilon, r}^{-}(r, s)=-\left(1+(\varepsilon-2 r) \kappa_{\varepsilon, r}^{+}\right)^{-1} \kappa_{\varepsilon, r}^{+}(r, s)$ since the distance between $\Gamma_{\varepsilon, r}^{+}$and $\Gamma_{\varepsilon, r}^{-}$is equal to $\varepsilon-2 r$. Finally, we denote by $s_{\varepsilon}$ the length of $\Gamma_{\varepsilon}^{+}$, that is $s_{\varepsilon}=\int_{\Gamma_{\varepsilon}^{+}} d s$, so that the curvilinear abscissa varies between 0 and $s_{\varepsilon}$. In the case of circular domains $\Omega_{\varepsilon}^{ \pm}=D\left(\mathbf{0}, R_{0} \pm \varepsilon / 2\right)$, the local curvature is $s$-independent and satisfies: $\kappa_{\varepsilon, r}^{ \pm}= \pm 1 /\left(R_{0} \pm \varepsilon / 2 \mp r\right)$ and $s_{\varepsilon}=2 \pi\left(R_{0}+\varepsilon / 2\right)$. Due to the complexity of the notations, we propose the following simplification. We first denote by $\kappa_{0}(s)$ the curvature at $\Gamma_{\varepsilon=0}^{+}$and by $h_{0}$ the scaling factor $h_{0}(r, s)=1+r \kappa_{0}(s)$. We deduce from the above simplification, that

$$
\kappa_{\varepsilon}^{ \pm}(s)= \pm h_{0}( \pm \varepsilon / 2, s)^{-1} \kappa_{0}(s), \quad \kappa_{\varepsilon, r}^{ \pm}(s)= \pm h_{0}( \pm(\varepsilon / 2-r), s)^{-1} \kappa_{0}(s)
$$

and

$$
h_{\varepsilon}^{ \pm}(r, s)=h_{0}( \pm(\varepsilon / 2-r), s)=1 \pm(\varepsilon / 2-r) \kappa_{0}(s) .
$$

We now present some important results about the factorization of operators, and symbolical expansions for the Dirichlet-to-Neumann map for the Schrödinger operator in imaginary-time. These computations are required to derive some accurate asymptotic estimates of the CSWR contraction factor.

\subsection{Nirenberg factorization and symbolic computation for the imaginary-time linear Schrödinger operator}

In $(r, s, t)$ local coordinates at the subdomain interface, the Schrödinger operator formally reads in imaginary-time

$$
P_{r}:=-\partial_{t}+\partial_{r}^{2}+\kappa \partial_{r}+h^{-1} \partial_{s}\left(h^{-1} \partial_{s}\right)-V_{r}(r) .
$$

In the definition of the operator $P_{r}$, the notations $\kappa(r, s)$ and $h(r, s)$ stand for $\kappa_{\varepsilon, r}^{ \pm}(s)$ and $h_{\varepsilon}^{ \pm}(r, s)$, respectively, and have to be specified depending on the considered subdomain/framework. At the interfaces, the operator $P_{r}$ can be formally factorized as follows. 
Proposition 3.1. The operators $P_{r}$ satisfies the following Nirenberg-like factorization

$$
P_{r}=\left(\partial_{r}+i \Lambda_{r}^{+}\left(r, s, t, \partial_{s}, \partial_{t}\right)\right)\left(\partial_{r}+i \Lambda_{r}^{-}\left(r, s, t, \partial_{s}, \partial_{t}\right)\right)+\mathcal{R},
$$

where $\mathcal{R} \in O P S^{-\infty}$ is a smoothing operator. The operators $\Lambda_{r}^{ \pm}$are pseudodifferential operators of order 1 (in time). Furthermore, their total symbols $\lambda_{r}^{ \pm}:=\sigma\left(\Lambda_{r}^{ \pm}\right)$can be expanded in $S_{S}^{1}$ as

$$
\lambda_{r}^{ \pm} \sim \sum_{j=0}^{+\infty} \lambda_{r, 1-j}^{ \pm},
$$

where $\lambda_{r, 1-j}^{ \pm}$are symbols corresponding to operators of order $1-j$. To simplify the notations, we omit here and hereafter the index $r$ in the latter symbols (i.e. $\lambda_{1-j}^{ \pm}$stands for $\lambda_{r, 1-j}^{ \pm}$).

The explicit expression of the symbols $\lambda_{r, 1-j}^{ \pm}$will be a keystone for establishing the convergence rate of the SWR method. We refer to [6] for the proof of this proposition in real time, and where a detailed construction of $\Lambda_{r}^{ \pm}$is iteratively established. In imaginary time, the proof is basically identical by replacing $\tau$ by $i \tau$. Let us remark that the definition of the operator $\Lambda_{r}^{ \pm}$is subdomain-dependent through $\kappa$ and $h$. Practically, the construction of $\Lambda_{r}^{ \pm}$is obtained through the computation of a finite number of elementary inhomogeneous symbols. For instance, one gets the following proposition, deduced from [6].

Proposition 3.2. Let us fix the principal symbol to

$$
\lambda_{1}^{+}=-\sqrt{-i \tau-h^{-2} \xi^{2}-V_{r}} .
$$

Then, the next symbol is given by

$$
\begin{aligned}
\lambda_{0}^{+}=-\frac{i}{2} \kappa & +\frac{i}{4} \frac{\left(\partial_{r} h^{-2}\right) \xi^{2}}{-i \tau-h^{-2} \xi^{2}+i h^{-1}\left(\partial_{s} h^{-1}\right) \xi-V_{r}} \\
& -\frac{i}{4} \frac{h^{-2}\left(\partial_{s} h^{-2}\right) \xi^{3}}{\sqrt{-i \tau-h^{-2} \xi^{2}+i h^{-1}\left(\partial_{s} h^{-1}\right) \xi-V_{r}}} .
\end{aligned}
$$

Any higher order elementary operators can also be constructed. In this formalism, one gets in each subdomain

$$
\lambda_{r}^{-}=-\lambda_{r}^{+}-i \kappa
$$

For a practical evaluation of the contraction factor, we have to evaluate the symbols at $r=0$. Using the simplified notations defined above, we identify $h(0, s)$ on $\Gamma_{\varepsilon}^{ \pm}$with $h_{\varepsilon}^{ \pm}(0, s)=1 \pm \varepsilon \kappa_{0}(s) / 2$ and $\kappa(0, s)$ with $\kappa_{\varepsilon}^{ \pm}(s)$ or equivalently $\pm h^{-1}(0, s) \kappa_{0}(s)$. Therefore, we deduce, from (19) on $\partial \Omega_{\varepsilon, r}^{ \pm}$, that we have at $r=0$

$$
\kappa(0, s)= \pm\left(1 \pm \varepsilon \kappa_{0}(s) / 2\right)^{-1} \kappa_{0}(s) .
$$

Then, a direct computation shows that

$$
\begin{aligned}
& h(0, s)=1 \pm \varepsilon \kappa_{0}(s) / 2, \quad \partial_{r} h^{-2}(0, s)= \pm \frac{2 \kappa_{0}(s)}{\left(1 \pm \varepsilon \kappa_{0}(s) / 2\right)^{3}} \\
& \partial_{s} h^{-2}(0, s)=\mp \frac{\varepsilon \partial_{s} \kappa_{0}(s)}{\left(1 \pm \varepsilon \kappa_{0}(s) / 2\right)^{3}} .
\end{aligned}
$$

Let us note that, for $\varepsilon=0$ (no overlap), we have $\kappa(0, s)= \pm \kappa_{0}(s)$ at $\Gamma_{\varepsilon}^{ \pm}, h(0, s)=1, \partial_{r} h^{-2}(0, s)= \pm 2 \kappa_{0}(s)$, and $\partial_{s} h^{-2}(0, s)=0$.

We now introduce the set of large enough frequencies $\tau(\tau \in \mathbb{R}$ and $|\tau| \gg 1)$, and which is denoted $\mathbb{R}_{\infty}$. In the following, suprema of contraction factors of the SWR algorithm will be restricted in the set. The fact that we consider $|\tau|$ large is a technical restriction coming from the use of Taylor's expansions, which 
will later be useful to derive simple expressions of the convergence rate of the SWR method. In fact, it is possible to partially relax this restriction by using Padé's approximants [6]. Notice however that, and as in the one-dimensional case, the contraction factors analytically derived below are good approximations of the numerical ones, see Section 4 as well [12].

The following symbols are defined [6] by replacing $\tau$ by $i \tau$ that

Proposition 3.3. At $r=0$, for $\varepsilon=0$ and for $j \geqslant 1$, the symbols $\lambda_{1-j}^{+}$, denoted by $\widetilde{\lambda}_{1-j}^{+}$at $\Gamma_{0}^{ \pm}$, are given by

$$
\begin{aligned}
\tilde{\lambda}_{1}^{+}= & -\sqrt{-i \tau-\xi^{2}-V_{0}}, \\
\tilde{\lambda}_{0}^{+}= & \mp \frac{i}{2} \kappa_{0} \pm \frac{i}{2} \frac{\kappa_{0} \xi^{2}}{-i \tau-\xi^{2}-V_{0}}, \\
\tilde{\lambda}_{-1}^{+}= & -\frac{1}{8} \frac{\kappa_{0}^{2}}{\sqrt{-i \tau-\xi^{2}-V_{0}}} \mp \frac{1}{2} \frac{\partial_{s} \kappa_{0} \xi}{-i \tau-\xi^{2}-V_{0}}-\frac{3}{4} \frac{\kappa_{0}^{2} \xi^{2}}{\sqrt{-i \tau-\xi^{2}-V_{0}}}, \\
& \mp \frac{1}{2} \frac{\partial_{s} \kappa_{0} \xi^{3}}{\left(-i \tau-\xi^{2}-V_{0}\right)^{2}}-\frac{5}{8} \frac{\kappa_{0}^{2} \xi^{4}}{\sqrt{-i \tau-\xi^{2}-V_{0}}} .
\end{aligned}
$$

Proof. Formulae (26) are obtained from [6] where we have replaced $\tau$ by $i \tau$.

From [6], we also have in the high frequency time regime.

Proposition 3.4. At $r=0$, for $\varepsilon=0$ and $\tau \in \mathbb{R}_{\infty}$, the symbols $\widetilde{\lambda}_{1-j}^{+}$are approximated up to a $\mathcal{O}\left(\tau^{-2}\right)$ by $\left(\widetilde{\lambda}_{1-j}^{+}\right)_{(-1)}$ given at $\Gamma_{0}^{ \pm}$by

$$
\begin{aligned}
& \left(\widetilde{\lambda}_{1}^{+}\right)_{(-1)}=e^{-i \pi / 4} \sqrt{-\tau}+e^{-i \pi / 4}\left(\frac{\xi^{2}}{2}+\frac{V_{0}}{2}\right) \frac{1}{\sqrt{-\tau}} \\
& \left(\widetilde{\lambda}_{0}^{+}\right)_{(-1)}=\mp \frac{i}{2} \kappa_{0} \pm \frac{1}{2} \frac{\kappa_{0} \xi^{2}}{\tau} \\
& \left(\widetilde{\lambda}_{-1}^{+}\right)_{(-1)}=-\frac{e^{-i \pi / 4}}{8} \frac{\kappa_{0}^{2}}{\sqrt{-\tau}} \mp \frac{i}{2} \frac{\partial_{s} \kappa_{0} \xi}{\tau} \\
& \left(\widetilde{\lambda}_{-2}^{+}\right)_{(-1)}=-\frac{1}{4} \frac{\partial_{\mathbf{n}} V_{0}}{\tau} \mp \frac{1}{8} \frac{\partial_{s}^{2} \kappa_{0}}{\tau} \mp \frac{1}{8} \frac{\kappa_{0}^{3}}{\tau}
\end{aligned}
$$

However, these results become incorrect for $\varepsilon>0$, that is when the subdomains overlap, and further computations are necessary. In particular, an explicit evaluation of $\lambda_{-1}^{+}$and $\lambda_{-2}^{+}$is necessary. The following result generalizes Proposition 3.4 to the case $\varepsilon>0$.

Proposition 3.5. For $\tau \in \mathbb{R}_{\infty}$, the symbols $\widetilde{\lambda}_{1-j}^{+}$are approximated at $\Gamma_{\varepsilon}^{ \pm}$, up to a $\mathcal{O}\left(\tau^{-2}\right)$, by

$$
\begin{aligned}
& \left(\widetilde{\lambda}_{1}^{+}\right)_{(-1)}=e^{-i \pi / 4} \sqrt{-\tau}+e^{-i \pi / 4}\left(\frac{h^{-2} \xi^{2}}{2}+\frac{V_{r}}{2}\right) \frac{1}{\sqrt{-\tau}} \\
& \left(\widetilde{\lambda}_{0}^{+}\right)_{(-1)}=-\frac{i}{2} \kappa+\frac{1 \kappa h^{-2} \xi^{2}}{\tau} \\
& \left(\widetilde{\lambda}_{-1}^{+}\right)_{(-1)}=-e^{-i \pi / 4} \frac{1}{4} \frac{\partial_{r} \kappa}{\sqrt{-\tau}}-e^{-i \pi / 4} \frac{1}{4} \frac{\kappa^{2}}{\sqrt{-\tau}}-i \partial_{s} \kappa \frac{h^{-2} \xi}{2 \tau}, \\
& \left(\widetilde{\lambda}_{-2}^{+}\right)_{(-1)}=-\frac{1 \partial_{\mathbf{n}} V_{r}}{4}-\frac{1 \partial_{s}^{2} \kappa}{8}-\frac{1}{8} \frac{\kappa^{3}}{\tau},
\end{aligned}
$$


where $\kappa, \partial_{r} \kappa, \partial_{s} \kappa$, and $\partial_{s}^{2} \kappa$ are defined in (74), (75), (76), (77). For $r=0$, the symbols (28) read as follows

$$
\begin{aligned}
& \left(\widetilde{\lambda}_{1}^{+}\right)_{(-1)}=e^{-i \pi / 4} \sqrt{-\tau}+e^{-i \pi / 4}\left(\frac{\left(1 \pm \varepsilon \kappa_{0} / 2\right)^{-2} \xi^{2}}{2}+\frac{V_{r}}{2}\right) \frac{1}{\sqrt{-\tau}}, \\
& \left(\widetilde{\lambda}_{0}^{+}\right)_{(-1)}=\mp \frac{i}{2\left(1 \pm \varepsilon \kappa_{0} / 2\right)} \kappa_{0} \pm \frac{1}{2\left(1 \pm \varepsilon \kappa_{0} / 2\right)^{2}} \frac{\kappa_{0} \xi^{2}}{\tau}, \\
& \left(\widetilde{\lambda}_{-1}^{+}\right)_{(-1)}=-\frac{e^{-i \pi / 4}}{8} \frac{\kappa_{0}^{2}}{\left(1 \pm \varepsilon \kappa_{0} / 2\right)^{2} \sqrt{-\tau}} \mp \frac{i}{2} \frac{\partial_{s} \kappa_{0} \xi}{\left(1 \pm \varepsilon \kappa_{0} / 2\right)^{2} \tau}, \\
& \left(\widetilde{\lambda}_{-2}^{+}\right)_{(-1)}=\mp \frac{1 \partial_{r} V_{r}}{4} \mp \frac{1}{8} \frac{\partial_{s}^{2} \kappa_{0}}{\tau} \mp \frac{1}{8} \frac{\kappa_{0}^{3}}{\tau\left(1 \pm \varepsilon \kappa_{0} / 2\right)^{3}} \mp \frac{1}{16} \frac{\varepsilon\left(\kappa_{0} \partial_{s}^{2} \kappa_{0}^{2}-\left(\partial_{s} \kappa_{0}\right)^{2}\right)}{\tau\left(1 \pm \varepsilon \kappa_{0} / 2\right)^{3}} .
\end{aligned}
$$

The proof which is rather technical is presented in Appendix.

We remark that, for $\varepsilon=0$, the approximate symbols coincide with the ones given in Proposition (3.4), since for $\varepsilon=0$ and on $\partial \Omega_{0}^{+}$,

$$
\kappa(0, s)=\kappa_{0}(s), \quad \partial_{s} \kappa(0, s)=\partial_{s} \kappa_{0}(s), \quad \partial_{s}^{2} \kappa(0, s)=\partial_{s}^{2} \kappa_{0}(s) .
$$

From these preliminary symbolic computations, it is possible to analyze the CSWR method as a fixed point method and to accurately determine its contraction factor.

\subsection{Asymptotic estimates of the contraction factor for the CSWR algorithm}

Assuming that $\psi^{\mp,(0)}$ are two given functions, the CSWR algorithm in cartesian coordinates, at iteration $k \geqslant 1$ reads as follows

$$
\left\{\begin{array}{l}
P \psi^{ \pm,(k)}=0, \text { in } \Omega_{\varepsilon}^{ \pm} \times \mathbb{R}_{+}^{*}, \\
\psi^{ \pm,(k)}(\cdot, 0)=\psi_{0}^{ \pm}, \text {in } \Omega_{\varepsilon}^{ \pm} \\
\psi^{ \pm,(k)}=\psi^{\mp,(k-1)}, \text { in } \Gamma_{\varepsilon}^{ \pm} \times \mathbb{R}_{+}^{*} .
\end{array}\right.
$$

For convenience, the CSWR algorithm will be analyzed in the system of coordinates $(r, s)$, that is denoting $\phi(r, s, t):=\psi(x(r, s), y(r, s), t)$ and $\phi_{0}(r, s)=\psi_{0}(x(r, s), y(r, s))$, we get:

$$
\begin{cases}P_{r} \phi^{ \pm,(k)} & =0, \text { in } \Omega_{\varepsilon}^{ \pm} \times \mathbb{R}_{+}^{*}, \\ \phi^{ \pm,(k)}(\cdot, 0) & =\phi_{0}^{ \pm}, \text {in } \Omega_{\varepsilon}^{ \pm}, \\ \phi^{ \pm,(k)}\left( \pm \varepsilon / 2, s_{0}, \cdot\right) & =\phi^{\mp,(k-1)}\left( \pm \varepsilon / 2, s_{0}, \cdot\right) \text { in } \mathbb{R}_{+}^{*} .\end{cases}
$$

We benefit from the fact that $\Gamma_{\varepsilon}^{+}$and $\Gamma_{\varepsilon}^{-}$are parallel at distance $\varepsilon$ to fix the curvilinear abscissa, $s_{0} \in\left[0, s_{\varepsilon}\right]$ in the transmission conditions at $\left( \pm \varepsilon / 2, s_{0}\right)$. Working with the error equations, i.e. $e_{P_{r}}^{C, \pm}$ corresponds to $\phi^{ \pm}$ for CSWR, we have by linearity in $\Omega_{\varepsilon}^{ \pm}$

$$
\begin{aligned}
& P_{r} e_{P_{r}}^{C, \pm}=0 \text { in } \Omega_{\varepsilon}^{ \pm} \times \mathbb{R}_{+}^{*}, \\
& e_{P_{r}}^{C, \pm}\left( \pm \varepsilon / 2, s_{0}, t\right)=h_{\left(\varepsilon, s_{0}\right)}^{ \pm}(t) \text { at }\left\{ \pm \varepsilon / 2, s_{0}\right\} \times \mathbb{R}_{+}^{*},
\end{aligned}
$$

where $P_{r}$ is given by (21). We use the index $P_{r}$ in $e_{P_{r}}^{C, \pm}$ to specify the operator to which the error is associated to, and the exponent $C$ stands for the CSWR algorithm. In the following, some other approximate errors are also used when the potential $V_{r}$ is variable. The time-dependent functions $h_{\left(\varepsilon, s_{0}\right)}^{ \pm}$are now assumed to be given. To lighten the notations $h_{\left(\varepsilon, s_{0}\right)}^{ \pm}$also denotes the extension of $h_{\left(\varepsilon, s_{0}\right)}^{ \pm}$to all $\mathbb{R}$, and which is null 
on $\mathbb{R}_{-}$. As proposed in [25] and [12], we want to determine the contraction factor $C_{P_{r}, \varepsilon}^{C}$ of $\mathcal{G}_{P_{r}}^{C 2}$ (setting $\mathcal{G}_{P_{r}}^{C 2}:=\mathcal{G}_{P_{r}}^{C} \circ \mathcal{G}_{P_{r}}^{C}$ ), where the mapping $\mathcal{G}_{P_{r}}^{C}$, with $s_{0} \in\left[0, s_{\varepsilon}\right]$ and $\varepsilon \in \mathbb{R}_{+}^{*}$, is defined by

$$
\mathcal{G}_{P_{r}}^{C}:\left\langle h_{\left(\varepsilon, s_{0}\right)}^{+}, h_{\left(\varepsilon, s_{0}\right)}^{-}\right\rangle \mapsto\left\langle e_{P_{r}}^{C,-}\left(\varepsilon / 2, s_{0}, \cdot\right), e_{P_{r}}^{C,+}\left(-\varepsilon / 2, s_{0}, \cdot\right)\right\rangle .
$$

To prove that $\mathcal{G}_{P_{r}}^{C 2}$ is a contraction, we can solve $(32)$ in $(r, s, \xi, \tau)$-coordinates exactly for constant $V_{r}$, only in the one-dimensional case [12]. For $V_{r} \neq 0$ (in fact, for a non constant potential $V_{r}$ ), we estimate the rate of convergence through approximations. Let us consider the general case with a potential $V_{r}$. According to [25], for a fixed time $T, \mathcal{G}_{P_{r}}^{C}$ is defined in $H_{0}^{3 / 4}(0, T)=\left\{\phi \in H^{3 / 4}(0, T): \phi(0,0,0)=0\right\}$. Let us characterize the part of the error $e_{P_{r}}^{C,+}$ (respectively $e_{P_{r}}^{C,-}$ ) which is a traveling wave in the overlapping region related to $\Omega_{\varepsilon}^{+}$(respectively $\Omega_{\varepsilon}^{-}$) domain $\mathbb{R}^{2} / \bar{\Omega}_{\varepsilon}^{+}$(respectively $\mathbb{R}^{2} / \bar{\Omega}_{\varepsilon}^{-}$). Therefore, we consider the system, for $\varepsilon>0$ and $s_{0} \in\left[0, s_{\varepsilon}\right]$,

$$
\left\{\begin{array}{l}
\left(\partial_{r}+i \Lambda_{r}^{\mp}\right) e_{\Lambda_{r}}^{C, \pm}=0, \text { in } \Omega_{\varepsilon}^{ \pm} \\
e_{\Lambda_{r}}^{C, \pm}(\cdot, \cdot, t)=h_{\left(\varepsilon, s_{0}\right)}^{ \pm}(t) \text { at }\left\{ \pm \varepsilon / 2, s_{0}\right\} \times \mathbb{R}
\end{array}\right.
$$

and $e_{\Lambda_{r}}^{C,+}$ (respectively $e_{\Lambda_{r}}^{C,-}$ ) must be understood as the outgoing (respectively incoming) part of $e_{P_{r}}^{C,+}$ (respectively $e_{P_{r}}^{C,-}$ ) through the boundary. As a consequence, the computation of $e_{\Lambda_{r}}^{C, \pm}$ provides an approximation of $e_{P_{r}}^{C, \pm}$ which is solution to $P_{r} e_{P_{r}}^{C, \pm}=0$, and we approximate $C_{P_{r}, \varepsilon}^{C}$ which is the contraction factor of $\mathcal{G}_{P_{r}}^{C 2}$ by $C_{\Lambda_{r}, \varepsilon}^{C}$ for $\mathcal{G}_{\Lambda_{r}}^{C 2}$, that is: $C_{P_{r}, \varepsilon}^{C} \approx C_{\Lambda_{r}, \varepsilon}^{C}$. For $V_{r}=0$, the solution to the first equation of system (34) can be made explicitly but only approximate through the Fourier transforms, $\mathcal{F}_{t, s}$ and $\mathcal{F}_{t}$ along the $(t, s)$ and $t$-directions (meaning at the symbol level). We define $\widehat{e}^{ \pm}=\mathcal{F}_{s, t}\left(e^{ \pm}\right)$and $\widehat{h}_{\left(\varepsilon, s_{0}\right)}^{ \pm}=\mathcal{F}_{(t, s)}\left(h_{\left(\varepsilon, s_{0}\right)}^{ \pm}\right)$. The solution to system $(34)$ is given in the $(r, s, \xi, \tau)$-space by

$$
\widehat{e}_{\Lambda_{r}}^{C, \pm}(r, s, \xi, \tau)=\widehat{h}_{\left(\varepsilon, s_{0}\right)}^{ \pm}(\tau) \exp \left(i \int_{ \pm \varepsilon / 2}^{r} \lambda_{r}^{\mp}\left(r^{\prime}, s, \xi, \tau\right) d r^{\prime}\right) .
$$

In addition, since we need to use the symbols for the imaginary-time equation, we obtain the correct symbols for (21) through the symbols defined in [6] for the Schrödinger equation, but with the following modifications: $t \rightarrow i t$ and $\tau \rightarrow i \tau$. If we define

$$
\mathcal{G}_{\Lambda_{r}}^{C}:\left\langle h_{\left(\varepsilon, s_{0}\right)}^{+}, h_{\left(\varepsilon, s_{0}\right)}^{-}\right\rangle \mapsto\left\langle e_{\Lambda_{r}}^{C,-}\left(\varepsilon / 2, s_{0}, \cdot\right), e_{\Lambda_{r}}^{C,+}\left(-\varepsilon / 2, s_{0}, \cdot\right)\right\rangle,
$$

we have the equalities

$$
\begin{aligned}
& \mathcal{F}_{t}\left(\mathcal{G}_{\Lambda_{r}}^{C 2}\left\langle h_{\left(\varepsilon, s_{0}\right)}^{+}(\tau), h_{\left(\varepsilon, s_{0}\right)}^{-}(\tau)\right\rangle\right) \\
& =\left\langle\mathcal{F}_{\xi}^{-1}\left(\exp \left(i \int_{-\varepsilon / 2}^{\varepsilon / 2}\left(\lambda^{-}\left(r^{\prime}, s^{\prime} \xi, \tau\right)-\lambda^{+}\left(r^{\prime}, s, \xi, \tau\right)\right) d r^{\prime}\right)\right)_{\mid s=s_{0}} \widehat{h}_{\left(\varepsilon, s_{0}\right)}^{+}(\tau),\right. \\
& \left.\quad \mathcal{F}_{\xi}^{-1}\left(\exp \left(i \int_{-\varepsilon / 2}^{\varepsilon / 2}\left(\lambda^{-}\left(r^{\prime}, s^{\prime}, \xi, \tau\right)-\lambda^{+}\left(r^{\prime}, s^{\prime}, \xi, \tau\right)\right) d r^{\prime}\right)\right)_{\mid s=s_{0}} \widehat{h}_{\left(\varepsilon, s_{0}\right)}^{-}(\tau)\right\rangle \\
& =\mathcal{F}_{\xi}^{-1}\left(\exp \left(\int_{-\varepsilon / 2}^{\varepsilon / 2} \kappa\left(r^{\prime}, s\right)-2 i \lambda^{+}\left(r^{\prime}, s, \xi, \tau\right) d r^{\prime}\right)\right)_{\mid s=s_{0}}\left\langle\widehat{h}_{\left(\varepsilon, s_{0}\right)}^{+}(\tau), \widehat{h}_{\left(\varepsilon, s_{0}\right)}^{-}(\tau)\right\rangle .
\end{aligned}
$$

We determine its contraction factor as a function of $\lambda_{r}^{+}$:

$$
\mathcal{F}_{t}\left(e_{\Lambda_{r}}^{C, \pm}\right)(r, s, \tau)=\widehat{h}_{\left(\varepsilon, s_{0}\right)}^{ \pm}(\tau) \mathcal{F}_{\xi}^{-1}\left(\exp \left(i \int_{ \pm \varepsilon / 2}^{r} \lambda_{r}^{ \pm}\left(r^{\prime}, s, \xi, \tau\right) d r^{\prime}\right)\right)
$$

Then, we write that

$$
\mathcal{F}_{t}\left(e_{\Lambda_{r}}^{C, \pm}\right)\left(\mp \varepsilon / 2, s_{0}, \tau\right)=\mathcal{F}_{t}\left(h_{\left(\varepsilon, s_{0}\right)}^{ \pm}(\tau)\right) \mathcal{F}_{\xi}^{-1}\left(\exp \left(i \int_{ \pm \varepsilon / 2}^{\mp \varepsilon / 2} \lambda_{r}^{ \pm}\left(r^{\prime}, s, \xi, \tau\right) d r^{\prime}\right)\right)_{\mid s=s_{0}}
$$


i.e.

$$
\begin{aligned}
\mathcal{F}_{t}\left\langle\mathcal{G}_{\Lambda_{r}}^{C 2}\left(h_{\left(\varepsilon / 2, s_{0}\right)}^{+}, h_{\left(\varepsilon, s_{0}\right)}^{-}\right)\right\rangle(\tau) \\
=\left\langle\mathcal{F}_{\xi}^{-1}\left(\exp \left(-i \int_{-\varepsilon / 2}^{\varepsilon / 2}\left(\lambda_{r}^{+}\left(r^{\prime}, s, \xi, \tau\right)-\lambda_{r}^{-}\left(r^{\prime}, s, \xi, \tau\right)\right) d r^{\prime}\right)\right)_{\mid s=s_{0}} \mathcal{F}_{t}\left(h_{\left(\varepsilon, s_{0}\right)}^{-}\right)(\tau),\right. \\
\left.\mathcal{F}_{\xi}^{-1}\left(\exp \left(-i \int_{\varepsilon / 2}^{-\varepsilon / 2}\left(\lambda_{r}^{+}\left(r^{\prime}, s, \xi, \tau\right)-\lambda_{r}^{-}\left(r^{\prime}, s, \xi, \tau\right)\right) d r^{\prime}\right)\right)_{\mid s=s_{0}} \mathcal{F}_{t}\left(h_{\left(\varepsilon, s_{0}\right)}^{+}\right)(\tau)\right\rangle .
\end{aligned}
$$

In addition, one gets

$$
\begin{aligned}
& \mathcal{F}_{t}\left\langle\mathcal{G}_{\Lambda_{r}}^{C 2}\left(h_{\left(\varepsilon, s_{0}\right)}^{+}, h_{\left(\varepsilon, s_{0}\right)}^{-}\right)\right\rangle(\tau) \\
& =\mathcal{F}_{\xi}^{-1}\left(\exp \left(-i \int_{-\varepsilon / 2}^{\varepsilon / 2}\left(\lambda_{r}^{+}\left(r^{\prime}, s, \xi, \tau\right)-\lambda_{r}^{-}\left(r^{\prime}, s, \xi, \tau\right)\right) d r^{\prime}\right)\right)_{\mid s=s_{0}} \\
& \times\left\langle\mathcal{F}_{t}\left(h_{\left(\varepsilon, s_{0}\right)}^{+}\right)(\tau), \mathcal{F}_{t}\left(h_{\left(\varepsilon, s_{0}\right)}^{-}\right)(\tau)\right\rangle \\
& =\mathcal{F}_{\xi}^{-1}\left(\exp \left(\int_{-\varepsilon / 2}^{\varepsilon / 2}\left(\kappa\left(r^{\prime}, s\right)-2 i \lambda_{r}^{+}\left(r^{\prime}, s, \xi, \tau\right)\right) d r^{\prime}\right)\right)_{\mid s=s_{0}} \\
& \times\left\langle\mathcal{F}_{t}\left(h_{\left(\varepsilon, s_{0}\right)}^{+}\right)(\tau), \mathcal{F}_{t}\left(h_{\left(\varepsilon, s_{0}\right)}^{-}\right)(\tau)\right\rangle .
\end{aligned}
$$

Let us introduce

$$
C_{\Lambda_{r}, \varepsilon}^{C}=\sup _{s_{0} \in\left[0, s_{\varepsilon}\right]} \sup _{\tau \in \mathbb{R}}\left|\mathcal{F}_{\xi}^{-1}\left(L_{\Lambda_{r}, \varepsilon}^{C}\right)_{\mid s=s_{0}}\right|
$$

where

$$
L_{\Lambda_{r}, \varepsilon}^{C}(\xi, \tau)=\left|\exp \left(-i \int_{-\varepsilon / 2}^{\varepsilon / 2}\left(\lambda_{r}^{+}\left(r^{\prime}, s, \xi, \tau\right)-\lambda_{r}^{-}\left(r^{\prime}, s, \xi, \tau\right)\right) d r^{\prime}\right)\right| .
$$

To evaluate this last term, we asymptotically expand $\lambda_{r}^{ \pm}$. The motivation is to simplify the expression of $L_{\Lambda_{r}, \varepsilon}^{C}$, in particular getting ride of the inverse Fourier transforms in $\xi$, and to have explicit expressions to work with. For $\tau \in \mathbb{R}_{\infty}$, and using the asymptotic expansion for LSE, applied in imaginary-time. We deduce that, following for instance [25] (and for a constant potential $V_{r}$ ), the contraction factors $C_{\Lambda_{r}, \varepsilon}^{C}$ of $\mathcal{G}_{\Lambda_{r}}^{C 2}$ and $C_{P_{r}, \varepsilon}^{C}$ of $\mathcal{G}_{P_{r}}^{C 2}$ are such that

$$
C_{P_{r}, \varepsilon}^{C} \approx C_{\Lambda_{r}, \varepsilon}^{C}=\sup _{s_{0} \in\left[0, s_{\varepsilon}\right]} \sup _{\tau \in \mathbb{R}}\left|\mathcal{F}_{\xi}^{-1}\left(L_{\Lambda_{r}, \varepsilon}^{C}(s, \xi, \tau)\right)_{\mid s=s_{0}}\right|
$$

where $L_{s, \Lambda_{r}, \varepsilon}^{C}(\xi, \tau)$ can only be approximately computed, as detailed below. We can then expect a fast convergence of the DDM at high frequency and/or for a large enough overlapping region of size $\varepsilon$. Note that the above approach is valid at any frequency, although the convergence will be naturally much slower for lowfrequency waves. Without overlap $(\varepsilon=0)$, as in the one-dimensional case, the CSWR algorithm diverges. However to get explicit contraction, factors additional approximations are necessary. More specifically, the computation of the contraction factor $C_{\Lambda_{r}, \varepsilon}^{C}$ of the associated mapping $\mathcal{G}_{\Lambda_{r}}^{C 2}$ requires the knowledge of the total symbols $\lambda_{r}^{ \pm}$. Unlike the one-dimensional case, even when $V_{r}$ is constant, this is generally not possible on non-circular domains. However, we have access to some asymptotic expansions $\left\{\lambda_{1-j}^{ \pm}\right\}_{j=0}^{+\infty}$ of $\lambda_{r}^{ \pm}$. To get such an estimate, we first expand $\lambda_{r}^{ \pm}$asymptotically as the sum of inhomogeneous symbols, where, again for notation convenience, we have omitted the index $r$ in the RHS $\left(\lambda_{1-j}^{ \pm}\right.$stands for $\left.\lambda_{r, 1-j}^{ \pm}\right)$:

$$
\lambda_{r}^{ \pm} \sim \sum_{j=0}^{ \pm \infty} \lambda_{1-j}^{ \pm}
$$


and then we truncate up to the $(p+1)$ first terms

$$
\lambda_{r}^{ \pm} \sim \lambda_{r}^{ \pm, p}=\sum_{j=0}^{p} \lambda_{1-j}^{ \pm}
$$

as proposed in [6]. This means that the approximate convergence rate is

$$
C_{P_{r}, \varepsilon}^{C} \approx C_{\Lambda_{r}, \varepsilon}^{C} \approx C_{\varepsilon}^{C, p}:=\sup _{s_{0} \in\left[0, s_{\varepsilon}\right]} \sup _{\tau \in \mathbb{R}}\left|\mathcal{F}_{\xi}^{-1}\left(L_{\varepsilon}^{C, p}(s, \xi, \tau)\right)_{\mid s=s_{0}}\right|,
$$

with

$$
L_{\varepsilon}^{C, p}(s, \xi, \tau)=\exp \left(i \int_{-\varepsilon / 2}^{\varepsilon / 2}\left(\lambda_{r}^{-, p}\left(r^{\prime}, s, \xi, \tau\right)-\lambda_{r}^{+, p}\left(r^{\prime}, s, \xi, \tau\right)\right) d r^{\prime}\right) .
$$

Let us recall now that if one chooses the principal symbol

$$
\lambda_{1}^{ \pm}=\mp \sqrt{-i \tau+h^{-2} \xi^{2}-V_{r}},
$$

then one gets for $p \geqslant 0$ :

$$
\lambda_{r}^{-, p}=-\lambda_{r}^{+, p}-i \kappa,
$$

implying that (39) becomes

$$
L_{\varepsilon}^{C, p}(s, \xi, \tau)=\exp \left(\int_{-\varepsilon / 2}^{\varepsilon / 2} \kappa\left(r^{\prime} s\right)-2 i \lambda_{r}^{+, p}\left(r^{\prime}, s, \xi, \tau\right) d r^{\prime}\right) .
$$

A third approximation step consists in developing each symbol $\lambda_{1-j}^{ \pm}, j=0, \ldots, p$, according to the small parameter $1 /|\tau|$, which means in the high time-frequency regime. More precisely, for each symbol $\lambda_{1-j}^{ \pm}$, we consider its Taylor's expansion $\left(\lambda_{1-j}^{ \pm}\right)_{1-p}$ up to the order $1 /|\tau|^{(p-1)}$

$$
\lambda_{r}^{ \pm, p} \sim \widetilde{\lambda}_{r}^{ \pm, p}=\sum_{j=0}^{p}\left(\widetilde{\lambda}_{1-j}^{ \pm}\right)_{(1-p)} .
$$

We then define

$$
\widetilde{L}_{\varepsilon}^{C, p}(s, \xi, \tau)=\exp \left(\int_{-\varepsilon / 2}^{\varepsilon / 2} \kappa\left(r^{\prime}, s\right)-2 i \widetilde{\lambda}_{r}^{+, p}\left(r^{\prime}, s, \xi, \tau\right) d r^{\prime}\right)
$$

and the associated high-frequency asymptotic convergence rate $\widetilde{C}_{\varepsilon}^{C, p}$ such that

$$
C_{P_{r}, \varepsilon}^{C} \approx C_{\Lambda_{r}, \varepsilon}^{C} \approx \widetilde{C}_{\varepsilon}^{C, p}:=\sup _{s_{0} \in\left[0, s_{\varepsilon}\right]} \sup _{\tau \in \mathbb{R}_{\infty}}\left|\mathcal{F}^{-1}\left(\widetilde{L}_{\varepsilon}^{C, p}(s, \xi, \tau)\right)_{\mid s=s_{0}}\right| .
$$

Let us set

$$
\begin{aligned}
& L_{\varepsilon, 1-j}(s, \xi, \tau)=\exp \left(\int_{-\varepsilon / 2}^{\varepsilon / 2} \kappa\left(r^{\prime}, s\right)-2 i \lambda_{1-j}^{+}\left(r^{\prime}, s, \xi, \tau\right) d r^{\prime}\right) \\
& \widetilde{L}_{\varepsilon, 1-j}^{p}(s, \xi, \tau)=\exp \left(\int_{-\varepsilon / 2}^{\varepsilon / 2} \kappa\left(r^{\prime}, s\right)-2 i\left(\widetilde{\lambda}_{1-j}^{+}\right)_{(1-p)}\left(r^{\prime}, s, \xi, \tau\right) d r^{\prime}\right) \mid .
\end{aligned}
$$

Then, we have

$$
L_{\varepsilon}^{C, p}=\prod_{j=0}^{p} L_{\varepsilon, 1-j} \text { and } \widetilde{L}_{\varepsilon}^{C, p}=\prod_{j=0}^{p} \widetilde{L}_{\varepsilon, 1-j}^{p} .
$$

This means that the elementary contribution of each inhomogeneous symbol and its approximate Taylorized symbol to the convergence rate can be studied separately, the global contribution being obtained by a simple multiplication. Based on these remarks, we now state some estimates of the rate of convergence of the CSWR algorithm for a general potential $V_{r}$. We can then prove the following result. 
Theorem 3.1. Let $V_{r}$ be a smooth spatial-dependent potential and let us assume that the symbols are defined as in Proposition 3.2. An asymptotic estimate of the contraction factor of the mapping $\mathcal{G}_{P_{r}}^{C 2}$ defined by (33), for the CSWR algorithm (58), is given by

$$
C_{P_{r}, \varepsilon}^{C} \approx C_{\varepsilon}^{C, 2}=\sup _{s_{0} \in\left[0, s_{\varepsilon}\right]} \sup _{\tau \in \mathbb{R}}\left|\mathcal{F}_{\xi}^{-1}\left(L_{\varepsilon}^{C, 2}(s, \xi, \tau)\right)_{\mid s=s_{0}}\right|,
$$

where

$$
L_{\varepsilon}^{C}(s, \xi, \tau) \approx L_{\varepsilon}^{C, 2}(s, \xi, \tau)=\prod_{j=0}^{2} L_{\varepsilon, 1-j}(s, \xi, \tau),
$$

and $L_{\varepsilon, 1-j}$ are given by (54), for $j=0,1,2$ and $\varepsilon>0$. In addition for $\tau \in \mathbb{R}_{\infty}$, one also gets the following approximation

$$
C_{P_{r}, \varepsilon}^{C} \approx \widetilde{C}_{\varepsilon}^{C, 2}=\sup _{s_{0} \in\left[0, s_{\varepsilon}\right]} \sup _{\tau \in \mathbb{R}_{\infty}}\left|\mathcal{F}_{\xi}^{-1}\left(\widetilde{L}_{\varepsilon}^{C, 2}(s, \xi, \tau)\right)_{\mid s=s_{0}}\right|
$$

where

$$
L_{\varepsilon}^{C}(s, \xi, \tau) \approx \widetilde{L}_{\varepsilon}^{C, 2}(s, \xi, \tau)=\prod_{j=0}^{2} \widetilde{L}_{\varepsilon, 1-j}^{p}(s, \xi, \tau)
$$

where $\widetilde{L}_{\varepsilon, 1-j}^{2}$ are given by (55), for $j=0,1,2,3$. More specifically, we get

$$
\begin{aligned}
C_{P_{r}, \varepsilon}^{C}= & \sup _{s_{0} \in\left[0, s_{\varepsilon}\right]} \sup _{\tau \in \mathbb{R}_{\infty}}\left\{|\tau|^{1 / 4} \frac{1}{\sqrt{2 \varepsilon}} \exp \left(-\frac{s_{0}^{2}}{8 \varepsilon} \sqrt{2|\tau|}\right)\right. \\
& \left.\quad \times \exp \left(-\varepsilon \sqrt{2|\tau|}+\varepsilon \frac{\kappa_{0}^{2}\left(s_{0}\right)}{2} \frac{1}{\sqrt{2|\tau|}}\right) \exp \left(-\frac{1}{\sqrt{2|\tau|}} \int_{-\varepsilon / 2}^{\varepsilon / 2} V_{r}\left(r^{\prime}, s_{0}\right) d r^{\prime}\right)\right\}
\end{aligned}
$$

In the case of polar symmetry (radial solution and circular interface), the approximate contraction factor is given by

$$
C_{P_{r}, \varepsilon}^{C} \approx \sup _{\tau \in \mathbb{R}_{\infty}}\left\{\exp \left(-\varepsilon \sqrt{2|\tau|}+\varepsilon \frac{\kappa_{0}^{2}}{2} \frac{1}{\sqrt{2|\tau|}}\right) \exp \left(-\frac{1}{\sqrt{2|\tau|}} \int_{-\varepsilon / 2}^{\varepsilon / 2} V_{r}\left(r^{\prime}\right) d r^{\prime}\right)\right\},
$$

where $\kappa_{0}$ is a constant, typically $1 / R_{0}$, if $R_{0}$ is the disc radius.

Notice that when the potential is positive, it confines the solution into the domain (standard situation for the GPE), then the convergence rate is improved. Again, in the non-overlapping case, the iterative method diverges. Remark that these contraction factors are consistent with the ones found in [12] in the one-dimensional case (take $\kappa_{0}=0$ ). Recall also that the approximate contraction factors are computed in a reduced frequency set $\mathbb{R}_{\infty}$. Despite this fact, we see in Section 4 and already noticed in [12] that they are in remarkable agreement with the contraction factors obtained from the full numerical experiments.

Proof. We first have

$$
\widehat{e}_{\Lambda_{r}}^{C, \pm}(r, s, \xi, \tau)=\widehat{h}_{\left(\varepsilon, s_{0}\right)}^{ \pm}(\tau) \exp \left(-i \int_{ \pm \varepsilon / 2}^{r} \lambda_{r}^{\mp}\left(r^{\prime}, s, \xi, \tau\right) d r^{\prime}\right) .
$$

This implies that

$$
\begin{aligned}
& \mathcal{F}_{t}\left(\mathcal{G}_{P_{r}}^{C} \circ \mathcal{G}_{P_{r}}^{C}\left\langle h_{\left(\varepsilon, s_{0}\right)}^{+}, h_{\left(\varepsilon, s_{0}\right)}^{-}\right\rangle\right) \\
& \approx \exp \left(i \int_{-\varepsilon / 2}^{\varepsilon / 2}\left(\lambda_{r}^{-}\left(r^{\prime}, s^{\prime}, \xi, \tau\right)-\lambda_{r}^{+}\left(r^{\prime}, s^{\prime}, \xi, \tau\right)\right) d r^{\prime}\right)\left\langle\widehat{h}_{\left(\varepsilon, s_{0}\right)}^{+}, \widehat{h}_{\left(\varepsilon, s_{0}\right)}^{-}\right\rangle .
\end{aligned}
$$


By using Proposition 3.2 for the imaginary-time equation, one gets

$$
\begin{aligned}
\lambda_{1}^{+}(r, s, \xi, \tau)= & -\sqrt{-i \tau-h^{-2} \xi^{2}-V_{r}}, \\
\lambda_{0}^{+}(r, s, \xi, \tau)= & -\frac{i}{2} \kappa\left(r^{\prime}, s\right)+\frac{i}{4} \frac{\left(\partial_{r} h^{-2}\right) \xi^{2}}{-i \tau-h^{-2} \xi^{2}+i h^{-1}\left(\partial_{s} h^{-1}\right) \xi-V_{r}} \\
& -\frac{i}{4} \frac{h^{-2}\left(\partial_{s} h^{-2}\right) \xi^{3}}{\sqrt{-i \tau-h^{-2} \xi^{2}+i h^{-1}\left(\partial_{s} h^{-1}\right) \xi-V_{r}}}
\end{aligned}
$$

and $\lambda_{r}^{-}=-\lambda_{r}^{+}-i \kappa$. Since $\kappa \in S_{S}^{0}$, then we have: $\lambda_{0}^{-}=-\lambda_{0}^{+}-i \kappa$, and $\lambda_{1-p}^{-}=-\lambda_{1-p}^{+}$for $p \in \mathbb{N}-\{1\}$. Therefore, a direct computation leads, for $p \in \mathbb{N}-\{1\}$, to

$$
L_{\varepsilon, p}(s, \xi, \tau)=\exp \left(\int_{-\varepsilon / 2}^{\varepsilon / 2}\left(-2 i \lambda_{1-p}^{+}\left(r^{\prime}, s, \xi, \tau\right)\right) d r^{\prime}\right)
$$

and

$$
L_{\varepsilon, 1}(s, \xi, \tau)=\exp \left(\int_{-\varepsilon / 2}^{\varepsilon / 2}\left(\kappa\left(r^{\prime}, s\right)-2 i \lambda_{0}^{+}\left(r^{\prime}, s, \xi, \tau\right)\right) d r^{\prime}\right)
$$

We note now that for $r \in[-\varepsilon / 2,0]$ then $\kappa(r, s)=-\left(1+(r-\varepsilon / 2) \kappa_{0}(s)\right)^{-1} \kappa_{0}(s)$ and for $r \in[0, \varepsilon / 2]$, $\kappa(r, s)=\left(1+(\varepsilon / 2-r) \kappa_{0}(s)\right)^{-1} \kappa_{0}(s)$. Based on this remark, we can estimate

$$
\exp \left(\int_{-\varepsilon / 2}^{\varepsilon / 2} \kappa\left(r^{\prime}, s\right) d r^{\prime}\right)
$$

(which is 1 for a flat boundary since $\kappa=0$ ). For a curved boundary, we obtain

$$
\begin{aligned}
& \exp \left(\int_{-\varepsilon / 2}^{\varepsilon / 2} \kappa\left(r^{\prime}, s\right) d r^{\prime}\right) \\
& =\exp \left(-\int_{-\varepsilon / 2}^{0}\left(1+\left(r^{\prime}-\varepsilon / 2\right) \kappa_{0}(s)\right)^{-1} \kappa_{0}(s) d r^{\prime}\right) \\
& \times \exp \left(\int_{0}^{\varepsilon / 2}\left(1+\left(\varepsilon / 2-r^{\prime}\right) \kappa_{0}(s)\right)^{-1} \kappa_{0}(s) d r^{\prime}\right) \\
& =\exp \left(-\varepsilon \kappa_{0}^{2}(s) \int_{0}^{\varepsilon / 2} \frac{1}{\left(1+\left(\varepsilon / 2-r^{\prime}\right) \kappa_{0}(s)\right)\left(1-\left(\varepsilon / 2+r^{\prime}\right) \kappa_{0}(s)\right)}\right) d r^{\prime}
\end{aligned}
$$

For $\varepsilon$ small enough, we have

$$
\begin{aligned}
& \exp \left(-\varepsilon \kappa_{0}^{2}(s) \int_{0}^{\varepsilon / 2} \frac{1}{\left(1+\left(\varepsilon / 2-r^{\prime}\right) \kappa_{0}(s)\right)\left(1-\left(\varepsilon / 2+r^{\prime}\right) \kappa_{0}(s)\right)}\right) d r^{\prime} \\
& \approx \exp \left(-\frac{\varepsilon^{2} \kappa_{0}^{2}(s)}{2\left(1-\varepsilon^{2} \kappa_{0}^{2}(s) / 4\right)}\right)
\end{aligned}
$$

and we deduce that in that case

$$
\exp \left(\int_{-\varepsilon / 2}^{\varepsilon / 2} \kappa\left(r^{\prime}, s\right) d r^{\prime}\right)<1
$$

However, interestingly this term does not have any impact on the SWR convergence, since one gets

$$
\kappa-2 i \lambda_{0}^{+}=\frac{1}{2} \frac{\left(\partial_{r} h^{-2}\right) \xi^{2}}{-i \tau-h^{-2} \xi^{2}+i h^{-1}\left(\partial_{s} h^{-1}\right) \xi-V_{r}}-\frac{1}{2} \frac{h^{-2}\left(\partial_{s} h^{-2}\right) \xi^{3}}{\sqrt{-i \tau-h^{-2} \xi^{2}+i h^{-1}\left(\partial_{s} h^{-1}\right) \xi-V_{r}^{2}}},
$$


i.e. the $\kappa$-term disappears from $\kappa-2 i \lambda_{r}^{+}$. Now let us analyze the contribution of the symbols to the convergence rate. According to (26), we have for instance

$$
\begin{aligned}
& \left.\left.L_{\varepsilon, 1}(s, \xi, \tau)=\exp \left(\int_{-\varepsilon / 2}^{\varepsilon / 2} 2 i \sqrt{-i \tau-h^{-2} \xi^{2}+V}\right)\right) d r^{\prime}\right), \\
& L_{\varepsilon, 0}(s, \xi, \tau)=\exp \left(-\frac{1}{2} \int_{-\varepsilon / 2}^{\varepsilon / 2} \frac{\left(\partial_{r} h^{-2}\right) \xi^{2}}{-i \tau-h^{-2} \xi^{2}+i h^{-1}\left(\partial_{s} h^{-1}\right) \xi-V_{r}} d r^{\prime}\right) \\
& \times \exp \left(-\frac{1}{2} \int_{-\varepsilon / 2}^{\varepsilon / 2} \frac{h^{-2}\left(\partial_{s} h^{-2}\right) \xi^{3}}{\sqrt{-i \tau-h^{-2} \xi^{2}+i h^{-1}\left(\partial_{s} h^{-1}\right) \xi-V_{r}}} d r^{\prime}\right) .
\end{aligned}
$$

Due to the complexity of the following coefficients, they are not fully reported. We however provide approximations that lead to precise estimates of their contributions in the SWR convergence. Formulae (54) are valid at any frequency. For large frequencies, expressions of the contraction factor can be simplified by using (28) in (52) and (53). In order to lighten the analysis while keeping the main feature of each of these terms, we assume that $\varepsilon$ is small (which is a reasonable assumption from the practical point of view) to first neglect the $O\left(\varepsilon^{3}\right)$-terms, then the $O\left(\varepsilon^{2}\right)$-terms. For $\tau \in \mathbb{R}_{\infty}$ and $\varepsilon \ll 1$, this means that we consider (29) in (52) and (53). Let us that, for $r \in[0, \varepsilon / 2]$, we have: $\kappa(r, s)=\left(1+(\varepsilon / 2-r) \kappa_{0}(s)\right)^{-1} \kappa_{0}(s)$ and, for $r \in[-\varepsilon / 2,0]$, $\kappa(r, s)=-\left(1-(\varepsilon / 2-r) \kappa_{0}(s)\right)^{-1} \kappa_{0}(s)$. Some basic algebraic computations lead to

$$
\begin{aligned}
& \widetilde{L}_{\varepsilon, 1}^{2}(s, \xi, \tau) \approx \exp \left(-2 e^{i \pi / 4} \varepsilon\left[\sqrt{-\tau}+\left(1+\varepsilon^{2} \kappa_{0}^{2}(s) / 4\right) \xi^{2} / 2\left(1-\varepsilon^{2} \kappa_{0}^{2}(s) / 4\right)^{2}\right]\right) \\
& \quad \times \exp \left(-e^{i \pi / 4} \int_{-\varepsilon / 2}^{\varepsilon / 2} V_{r}\left(r^{\prime}, s_{0}\right) d r^{\prime} / \sqrt{-\tau}\right) \\
& \approx \exp \left(-e^{i \pi / 4} \varepsilon\left[2 \sqrt{-\tau}+\xi^{2} / \sqrt{-\tau}\right]\right) \exp \left(-e^{i \pi / 4} \int_{-\varepsilon / 2}^{\varepsilon / 2} V_{r}\left(r^{\prime}, s_{0}\right) d r^{\prime} / \sqrt{-\tau}\right), \\
& \widetilde{L}_{\varepsilon, 0}^{2}(s, \xi, \tau) \approx \exp \left(-i \varepsilon^{2} \kappa_{0}^{2}(s) \xi^{2} /\left[\tau\left(1-\varepsilon^{2} \kappa_{0}^{2}(s) / 4\right)^{2}\right]\right) \approx 1, \\
& \widetilde{L}_{\varepsilon,-1}^{2}(s, \xi, \tau) \approx \exp \left(2 e^{i \pi / 4} \varepsilon\left[\kappa_{0}^{2}(s)\left(1+\varepsilon^{2} \kappa_{0}^{2}(s) / 4\right) /\left(8 \sqrt{-\tau}\left(1-\varepsilon^{2} \kappa_{0}(s) / 4\right)^{2}\right)\right]\right)+ \\
& \times \exp \left(\varepsilon^{2}\left[\left(-\xi \partial_{s} \kappa_{0}(s)+\left(\partial_{s} \kappa_{0}^{2}(s)-2 \kappa_{0}^{2}(s)\right)\left(1+\varepsilon^{2} \kappa_{0}^{2}(s) / 4\right) / 4\right) /\left(2 \tau\left(1-\varepsilon^{2} \kappa_{0}^{2}(s) / 4\right)^{2}\right)\right]\right) \\
& \quad \approx \exp \left(\varepsilon e^{i \pi / 4} \kappa_{0}^{2}(s) / 4 \sqrt{-\tau}\right), \\
& \widetilde{L}_{\varepsilon,-2}^{2}(s, \xi, \tau) \approx 1 .
\end{aligned}
$$

Then, one gets

$$
L_{\varepsilon}^{C, 2}=\prod_{j=0}^{2} L_{\varepsilon, 1-j} \text { and } \widetilde{L}_{\varepsilon}^{C, 2}=\prod_{j=0}^{2} \widetilde{L}_{\varepsilon, 1-j}^{2}
$$

and

$$
\widetilde{C}_{\varepsilon}^{C, 2}:=\sup _{s_{0} \in\left[0, s_{\varepsilon}\right]} \sup _{\tau \in \mathbb{R}_{\infty}}\left|\mathcal{F}_{\xi}^{-1}\left(\widetilde{L}_{\varepsilon}^{C, 2}(s, \xi, \tau)\right)_{\mid s=s_{0}}\right| .
$$

Now, we recall that $\kappa$ is domain dependent (positive in $\Omega_{\varepsilon}^{+}$and negative in $\Omega_{\varepsilon}^{-}$). To be more explicit about the convergence rate, we recall that for $\alpha \in \mathbb{C}^{*}$

$$
\mathcal{F}_{\xi}^{-1}\left(\exp \left(-\alpha \xi^{2}\right)\right)_{\mid s=s_{0}}=\frac{1}{\sqrt{2 \alpha}} \exp \left(-\frac{s_{0}^{2}}{4 \alpha}\right) .
$$

An explicit expression of the contraction factor can be provided by using (55) and estimating $\mathcal{F}_{\xi}^{-1}\left(\prod_{j=0}^{3} \widetilde{L}_{\varepsilon, 1-j}^{2}\right)_{\mid s=s_{0}}$. We set

$$
\left.\alpha_{\varepsilon}(\tau)=\varepsilon e^{i \pi / 4} \frac{1}{\sqrt{-\tau}}, \quad \beta_{\varepsilon}(\tau)=-2 e^{i \pi / 4} \varepsilon\left[\sqrt{-\tau}-\frac{\kappa_{0}^{2}(s)}{4 \sqrt{-\tau}}\right]-e^{i \pi / 4} \frac{\int_{-\varepsilon / 2}^{\varepsilon / 2} V_{r}\left(r^{\prime}, s_{0}\right) d r^{\prime}}{\sqrt{-\tau}}\right]
$$


and deduce that, for $\varepsilon>0$,

$$
\begin{aligned}
\mathcal{F}_{\xi}^{-1}\left(\prod_{j=0}^{3} \widetilde{L}_{\varepsilon, 1-j}^{2}\right)_{\mid s=s_{0}} \approx \frac{1}{\sqrt{2 \alpha_{\varepsilon}(\tau)}} \exp \left(\beta_{\varepsilon}(\tau)\right) \exp \left(-\frac{s_{0}^{2}}{4 \alpha_{\varepsilon}(\tau)}\right) \\
=(-\tau)^{1 / 4} \frac{e^{-i \pi / 8}}{\sqrt{2 \varepsilon}} \exp \left(-\frac{s_{0}^{2} e^{-i \pi / 4}}{4 \varepsilon} \sqrt{-\tau}\right) \\
\quad \times \exp \left(-2 e^{i \pi / 4} \varepsilon\left(\sqrt{-\tau}-\frac{\kappa_{0}^{2}\left(s_{0}\right)}{4 \sqrt{-\tau}}\right)\right) \exp \left(-e^{i \pi / 4} \frac{\int_{-\varepsilon / 2}^{\varepsilon / 2} V_{r}\left(r^{\prime}, s_{0}\right) d r^{\prime}}{\sqrt{-\tau}}\right) .
\end{aligned}
$$

From this last calculation, we can write that

$$
\begin{aligned}
\left|\mathcal{F}_{\xi}^{-1}\left(\prod_{j=0}^{3} \widetilde{L}_{\varepsilon, 1-j}^{2}\right)_{\mid s=s_{0}}\right| & \approx|\tau|^{1 / 4} \frac{1}{\sqrt{2 \varepsilon}} \exp \left(-\frac{s_{0}^{2}}{8 \varepsilon} \sqrt{2|\tau|}\right) \\
& \quad \times \exp \left(-\varepsilon \sqrt{2|\tau|}+\varepsilon \frac{\kappa_{0}^{2}\left(s_{0}\right)}{2} \frac{1}{\sqrt{2|\tau|}}\right) \exp \left(-\frac{1}{\sqrt{2|\tau|}} \int_{-\varepsilon / 2}^{\varepsilon / 2} V_{r}\left(r^{\prime}, s_{0}\right) d r^{\prime}\right) .
\end{aligned}
$$

We deduce the convergence of the CSWR-DDM for positive $\varepsilon$. Furthermore, the rate of convergence is estimated by

$$
\begin{aligned}
C_{P_{r}, \varepsilon}^{C} \approx C_{\varepsilon}^{C, 2} & \approx \sup _{s_{0} \in\left[0, s_{\varepsilon}\right]} \sup _{\tau \in \mathbb{R}_{\infty}}\left\{|\tau|^{1 / 4} \frac{1}{\sqrt{2 \varepsilon}} \exp \left(-\frac{s_{0}^{2}}{8 \varepsilon} \sqrt{2|\tau|}\right)\right. \\
& \left.\times \exp \left(-\varepsilon \sqrt{2|\tau|}+\varepsilon \frac{\kappa_{0}^{2}\left(s_{0}\right)}{2} \frac{1}{\sqrt{2|\tau|}}\right) \exp \left(-\frac{1}{\sqrt{2|\tau|}} \int_{-\varepsilon / 2}^{\varepsilon / 2} V_{r}\left(r^{\prime}, s_{0}\right) d r^{\prime}\right)\right\} .
\end{aligned}
$$

Finally, in polar symmetry, the term $|\tau|^{1 / 4} \frac{1}{\sqrt{2 \varepsilon}} \exp \left(-\frac{s_{0}^{2}}{8 \varepsilon} \sqrt{2|\tau|}\right)$ is not present in the contraction factor, as this contribution actually comes from the inverse Fourier transform in $\xi$ of the coefficient $\exp (-$ $\left.e^{i \pi / 4} \varepsilon \xi^{2} / \sqrt{-\tau}\right)$ in $\widetilde{L}_{\varepsilon, 1}^{2}(55)$.

Remark: Asymptotic estimates of the contraction factor for OSWR algorithm. Let us remark that a similar analysis can be applied to more general SWR, such as the Optimized Schwarz Waveform Relaxation (OSWR) method [32], where transmission boundary conditions are imposed, based on absorbing boundary operators. More specifically, if we assume that $\phi^{ \pm,(0)}\left(\mp \varepsilon / 2, s_{0}, \cdot\right)$ and $\phi_{0}^{ \pm}$are some given functions, then the OSWR algorithm, at iteration $k \geqslant 1$, reads as follows

$$
\left\{\begin{array}{l}
P_{r} \phi^{ \pm,(k)}=0, \text { in } \Omega_{\varepsilon}^{ \pm} \times \mathbb{R}_{+}^{*}, \\
\phi^{ \pm,(k)}(\cdot, 0)=\phi_{0}^{ \pm}, \text {in } \Omega_{\varepsilon}^{ \pm}, \\
\left(\partial_{r}+i \Lambda_{r}^{ \pm, p}\right) \phi^{ \pm,(k)}\left( \pm \varepsilon / 2, s_{0}, \cdot\right)=\left(\partial_{r}+i \Lambda_{r}^{ \pm, p}\right) \phi^{\mp,(k-1)}\left( \pm \varepsilon / 2, s_{0}, \cdot\right) \text { in } \mathbb{R}_{+}^{*} .
\end{array}\right.
$$

The convergence analysis of two-dimensional OSWR for LSE and NLSE uses some similar tools and ideas as above and will be presented in a forthcoming paper.

\subsection{Wellposedness of the CSWR algorithm}

We now study the well-posedness of the CSWR algorithm with smooth interface. This well-posedness result is a consequence of the first trace theorem for parabolic problem [34].

$$
\left\{\begin{array}{l}
\partial_{t} \phi^{ \pm}-\triangle \phi^{ \pm}+V(x, y) \phi^{ \pm}=0 \text { in } \Omega_{\varepsilon}^{ \pm} \times(0, T) \\
\phi^{ \pm}(\cdot, 0)=\phi_{0} \text { in } \Omega_{\varepsilon}^{ \pm} \\
\phi^{ \pm}(\cdot, \cdot)=g(\cdot, \cdot) \text { in } \Gamma_{\varepsilon}^{ \pm} \times(0, T)
\end{array}\right.
$$


We recall that from [34], for $\phi_{0} \in H^{1}(\Omega), V \in C_{0}^{\infty}(\Omega)$, and $g \in H^{3 / 2,3 / 4}(\Gamma \times(0, T))$ there exists a unique solution $\phi \in H^{2,1}(\Omega \times(0, T))$ such that

$$
\left\{\begin{array}{l}
\partial_{t} \phi-\triangle \phi+V(x, y) \phi=0 \text { in } \Omega \times(0, T) \\
\phi(\cdot, 0)=\phi_{0} \text { in } \Omega \\
\phi(\cdot, \cdot)=g(\cdot, \cdot) \text { in } \Gamma \times(0, T)
\end{array}\right.
$$

with the compatibility condition $g(\cdot, 0)=\phi_{0}(\cdot)$, on $\Gamma$.

From this result, we can easily construct a sequence of iterates $\phi^{ \pm,(k)}$. Let us first, show the existence of weak solutions on each subdomain. We again assume that $\phi_{0}$ is in $H^{1}(\Omega)$, and $g_{\varepsilon}^{ \pm} \in H^{3 / 2,3 / 4}\left(\Gamma_{\varepsilon}^{ \pm} \times(0, T)\right)$. From the above result, there exists a unique solution $\phi^{ \pm} \in H^{2,1}(\Omega \times(0, T))$ with $g_{\varepsilon}^{ \pm}(\cdot, 0)=\phi_{0}$ on $\Gamma_{\varepsilon}^{ \pm}$. In order to construct a sequence of iterates $\phi^{ \pm,(k)}$, it is necessary that on $\Gamma_{\varepsilon}^{ \pm} \times(0, T), \phi^{ \pm} \in H^{3 / 2,3 / 4}\left(\Gamma_{\varepsilon}^{ \pm} \times(0, T)\right)$. We then need to show that for $\phi^{ \pm} \in H^{2,1}\left(\Omega_{\varepsilon}^{ \pm} \times(0, T)\right)$, its trace on $\Gamma_{\varepsilon}^{ \pm}$is in the apropriate space. From the first trace theorem in [34], $\phi^{ \pm,(k-1)} \in H^{2,1}\left(\Omega_{\varepsilon}^{ \pm}\right)$then on $\Gamma_{\varepsilon}^{ \pm}, \phi^{ \pm}$belongs to $H^{3 / 2,3 / 4}\left(\Gamma_{\varepsilon}^{ \pm} \times(0, T)\right)$ which is exactly the needed regularity. Hence a sequence of iterates can be constructed. We have then

Theorem 3.2. Assuming that $\phi_{0} \in H^{1}(\Omega), V$ is smooth, $\Gamma_{\varepsilon}^{ \pm}$are smooth curves then, the CSWR iterates $\left(\phi^{-,(k)}, \phi^{+,(k)}\right)$ defined in (4) with $B^{ \pm}=I$ d, exist in $\left(H^{2,1}\left(\Omega_{\varepsilon}^{ \pm} \times(0, T)\right)\right)^{2}$.

\subsection{Convergence of the CSWR algorithm}

We can now state the convergence theorem for the overall CNGF-SWR method. From the evaluation of the contraction factor and by using a similar analysis as in [25], we can deduce an asymptotic convergence result (Theorem 3.3) following the same strategy as Section 2.4 in [12]. At any Schwarz iteration $k$, we denote by $T^{(k)}$ the convergence time of the CNGF algorithm thanks to the stopping criterion: $\phi(\cdot, t)=\phi\left(\cdot, T^{(k)}\right)$, for any $t \geqslant T^{(k)}$. In practice, we introduce a positive parameter $\delta$ and, at Schwarz iteration $k$, the imaginary-time iterations are stopped when, for $n \geqslant 0$, one gets

$$
\left\|\phi^{ \pm,(k)}\left(\cdot, t_{n+1}^{-}\right)-\phi^{ \pm,(k)}\left(\cdot, T^{(k)}\right)\right\|_{L^{\infty}\left(\mathbb{R}^{2}\right)} \leqslant \delta .
$$

To prove the result, we assume that the sequence of stopping times $\left\{T^{(k)}\right\}_{k}$ i) satisfies $T^{(k)} \leqslant T^{(k-1)}$ (at least for $k$ large enough) and ii) is convergent to $T^{\left(k^{\text {cvg }}\right)}>0$. This last assumption is morally reasonable and is confirmed numerically both in the one- (see [12]) and two-dimensional settings (see Section 4). It means that the larger the iteration $k$, the faster the CNGF algorithm to reach the stationary state. By extension of Theorem 5.8 in [25], we can directly adapt Theorem 2.7 in [12]:

Theorem 3.3. Let us assume that i) $V_{r}$ is a smooth and bounded radial dependent function, ii) the sequence $\left\{T^{(k)}\right\}_{k}$ is decreasing and convergent to $T^{(c v g)}>0$, i.e. there exists $k_{0}$ such that $0<T^{(c v g)} \leqslant T^{(k)} \leqslant T^{(k-1)}$ for all $k \geqslant k_{0}$, with $\lim _{k \rightarrow+\infty} T^{(k)}=T^{(\mathrm{cvg})}$, and iii) $T^{\left(k_{0}\right)}$ is finite. Then, the following inequalities hold

$$
\left\|e_{\Lambda_{r}}^{C, \pm}\right\|_{L^{2}\left(\mathbb{R}_{+} ; H^{2}\left(\Omega_{\varepsilon}^{ \pm}\right)\right)} \leqslant C_{\Lambda_{r}, \varepsilon}^{C}\left\|h_{\varepsilon}^{ \pm}\right\|_{\left(H^{3 / 4}\left(\mathbb{R}_{+}\right)\right)^{2}}
$$

and

$$
\begin{aligned}
\left\|\left(\left(e_{\Lambda_{r}}^{C,+}\right)^{2 k+1},\left(e_{\Lambda_{r}}^{C,-}\right)^{2 k+1}\right)\right\|_{H^{2,1}\left(\Omega_{\varepsilon}^{+} \times\left(0, T^{\left(k_{0}\right)}\right)\right) \times H^{2,1}\left(\Omega_{\varepsilon}^{-} \times\left(0, T^{\left(k_{0}\right)}\right)\right)} & \\
& \leqslant D\left(C_{\Lambda_{r}, \varepsilon}^{C}\right)^{k}\left\|\left(h_{\varepsilon}^{+, 0}, h_{\varepsilon}^{-, 0}\right)\right\|_{\left(H^{3 / 4}\left(0, T^{\left(k_{0}\right)}\right)\right)^{2}},
\end{aligned}
$$

where $D$ is a constant, and starting from a null initial guess in $\Omega_{\varepsilon}^{ \pm}$. The positive real-valued constant $C_{\Lambda_{r}, \varepsilon}^{C}$ is defined as the contraction factor of the mapping $\mathcal{G}_{\Lambda_{r}}^{C, p 2}$.

The extension of the result for the LSE can also be stated for the GPE. More specifically, the result holds for $k$ large enough or for $\phi_{0}$ sufficiently close to an eigenfunction, denoted by $\phi_{s}$. Indeed, in both cases, the function $\phi^{(k)}$ is close to an eigenstate and, as a consequence, the nonlinearity $\nu\left|\phi^{(k)}(\cdot, t)\right|^{2}$ is expected 
to behave almost like a fixed linear potential. In other words, from (47), we asymptotically expect that the contraction factor for CSWR, denoted by $C_{\varepsilon}^{\mathrm{GP}, C}$, behaves for $\varepsilon$ small enough as

$$
\begin{aligned}
C_{\varepsilon}^{\mathrm{GP}, C} \approx \widetilde{C}_{\varepsilon}^{\mathrm{GP}, C, 2}(\tau, s):= & \left\{|\tau|^{1 / 4} \frac{1}{\sqrt{2 \varepsilon}} \exp \left(-\frac{s^{2}}{8 \varepsilon} \sqrt{2|\tau|}\right)\right. \\
& \times \exp \left(-\varepsilon \sqrt{2|\tau|}+\varepsilon \frac{\kappa_{0}^{2}(s)}{4} \frac{1}{\sqrt{2|\tau|}}\right) \\
& \left.\times \exp \left(-\frac{\varepsilon}{\sqrt{2|\tau|}} \int_{-\varepsilon / 2}^{\varepsilon / 2} V_{r}\left(r^{\prime}, s\right)+\nu\left|\phi_{s}\left(r^{\prime}, s\right)\right|^{2} d r^{\prime}\right)\right\} .
\end{aligned}
$$

\subsection{Remark about the convergence of CSWR method in real time}

The technique which is exposed in this paper, can in principle be extended to real time. In this goal, we first have to define the $E$-quasi hyperbolic, elliptic and glancing zones [7], with $E=(1,2)$.

Definition 3.1. We define the E-quasi hyperbolic zone of the Schrödinger operator as the set $\mathcal{H}^{(s)}$ of points $(s, t, \xi, \tau)$ such that

$$
\mathcal{H}^{(s)}=\left\{(s, t, \xi, \tau): \tau+\xi^{2}+V<0\right\}
$$

Let us remark that the construction of the transmission conditions for the real-time dynamics is then realized under the microlocal assumption that the points $(s, t, \xi, \tau)$ lie in $\mathcal{H}^{(s)}$. This hypothesis characterizes the propagative part of the wave. Two other regions can be also defined: the $E$-quasi elliptic zone $\mathcal{E}^{(s)}$ given by

$$
\mathcal{E}^{(s)}=\left\{(s, t, \xi, \tau): \tau+\xi^{2}+V>0\right\}
$$

which gives the evanescent (exponentially decaying) part of the wave and the E-quasi glancing zone which is the complementary set $\mathcal{G}^{(s)}$ of $\mathcal{E}^{(s)} \cup \mathcal{H}^{(s)}$. This last region is reduced to $\{0\}$ if $u$ is not tangentially incident to $\Sigma$. In real-time, we usually assume that the frequencies are defined in $\mathcal{H}^{(s)}$. This assumption is not always valid but is true if we suppose that the evanescent part is reduced to $\{0\}$. In imaginary-time $(\tau \rightarrow i \tau)$, these zones are empty. In principle, we can then study the convergence of the SWR-DDM in real time, by replacing $\tau \rightarrow i \tau$ and $t \rightarrow i t$ in the above symbols, and analyzing the corresponding contraction factors respectively zones. According to [32], a finer approximation of the solution to (34) (in real time), could however be necessary to get an accurate estimation of the contraction factor in the hyperbolic zone.

Such an analysis is currently under inverstigation, in the one-dimensional case for the linear Schrödinger equation with non-constant coefficients.

\section{Numerical examples}

This section is dedicated to some numerical experiments illustrating the Theorems 3.1 and 3.3.

\subsection{Numerical examples in the two-dimensional case with polar symmetry}

We first consider a problem with polar symmetry, which allows for searching for a $s$-independent solution. The Hamiltonian operator reads

$$
H_{r} \phi(r, t)=\left(-\partial_{r}^{2}-\frac{1}{r} \partial_{r}+V(r)+\nu|\phi(r, t)|^{2}\right) \phi(r, t),
$$

where $V_{r}$ is the radial-dependent potential, and $\nu>0$. Although simple, this test proposes an illustration of the curvature effect on the CSWR convergence rate. More specifically, we expect to numerically validate (51). To this end, we consider the following circular domains: $\Omega_{R_{0}, \varepsilon}^{+}=D\left(0, R_{0}+\varepsilon / 2\right)$ and $\Omega_{R_{0}, \varepsilon}^{-}=$ $D\left(0, R_{1}\right)-D\left(0, R_{0}-\varepsilon / 2\right)$ with $R_{1}>R_{0}$. Dirichlet transmission conditions are imposed at $r= \pm \varepsilon / 2$, 
and null Dirichlet boundary conditions are set at $r=R_{1}$. The data of the problem are as follows: we set $R_{1}=4 / 5, R_{0}=2 / 5$ and

$$
V(r)=100\left(r-R_{0}\right)^{4}, \phi_{0}(r)=\frac{e^{-100\left(r-R_{0}\right)^{2}}}{\left\|e^{-100\left(r-R_{0}\right)^{2}}\right\|_{L^{2}\left(D\left(0, R_{1}\right)\right.}} .
$$

This choice is motivated by the need to enhance the curvature-related effect of $1 / R_{0}^{2}$ on the rate of convergence; the smaller $R_{0}$, the larger the curvature and the higher the effect on the convergence rate. In particular, we will observe that the convergence rate of the SWR method is slowed down by a curvature effect. An semi-implicit Euler (SIE) scheme is used to approximate the Schrödinger equation in polar coordinates with polar symmetry. The CSWR algorithm reads, for $n \geqslant 0$,

$$
\left\{\begin{array}{l}
\left(\frac{I}{\Delta t}-\partial_{r}^{2}-\frac{1}{r} \partial_{r}+V(r)+\nu\left|\phi^{ \pm, n,(k)}\right|^{2}\right) \widetilde{\phi}^{ \pm, n+1,(k)}=\frac{\phi^{ \pm, n,(k)}}{\Delta t}, \text { in } \Omega_{R_{0}, \varepsilon}^{ \pm}, \\
\widetilde{\phi}_{ \pm \varepsilon, n+1,(k)}^{ \pm}=\widetilde{\phi}_{ \pm \varepsilon / 2}^{\mp, n+1,(k-1)} \\
\widetilde{\phi}^{+, n+1,(k)}=0, \text { at } r=R_{1},
\end{array}\right.
$$

where $\widetilde{\phi}_{ \pm \varepsilon / 2}^{ \pm, n+1,(k)}$ denotes $\widetilde{\phi}^{ \pm, n+1,(k)}$ at $R_{0} \pm \varepsilon / 2$. At each iteration $(n+1, k)$ the global solution $\widetilde{\phi}^{n+1,(k)}$ needs to be normalized

$$
\phi^{n+1,(k)}:=\frac{\widetilde{\phi}^{+, n+1,(k)}+\widetilde{\phi}^{-, n+1,(k)}}{\left\|\widetilde{\phi}^{+, n+1,(k)}+\widetilde{\phi}^{-, n+1,(k)}\right\|_{L^{2}\left(D\left(0, R_{1}\right)\right)}} .
$$

At any Schwarz iteration, the CNGF method tolerance is fixed to $\delta=10^{-12}$, i.e.

$$
\left\|\phi^{n+1,(k)}-\phi^{n,(k)}\right\|_{\infty} \leqslant \delta
$$

where $\|\psi\|_{\infty}:=\sup _{r \in D\left(0, R_{1}\right)}|\psi(r)|$. When the convergence is reached, then the stopping time is such that: $T^{(k)}:=T^{\mathrm{cvg},(k)}=n^{\mathrm{cvg},(k)} \Delta t$ for a converged solution $\phi^{\mathrm{cvg},(k)}$ reconstructed from the two subdomains solutions $\phi^{ \pm, \mathrm{cvg},(k)}$. The convergence criterion for the Schwarz DDM is set to

$$
\|\| \phi_{\mid \Gamma_{\varepsilon}}^{+, \mathrm{cvg},(k)}-\phi_{\mid \Gamma_{\varepsilon}}^{-, \mathrm{cvg},(k)}\left\|_{\infty, \Gamma_{\varepsilon}}\right\|_{L^{2}\left(0, T^{\left(k^{\mathrm{cvg}}\right)}\right)} \leqslant \delta^{\mathrm{Sc}},
$$

with $\delta^{\mathrm{Sc}}=10^{-14}$ ("Sc" for Schwarz). When the convergence of the whole iterative algorithm is obtained at Schwarz iteration $k^{\text {cvg }}$, then one gets the converged global solution $\phi^{\text {cvg }}:=\phi^{\text {cvg, }\left(k^{\text {cvg }}\right)}$ in $D\left(0, R_{1}\right)$. In this example the curvature on $\Gamma_{\varepsilon}^{ \pm}$is given by $1 /\left(R_{0} \pm \varepsilon / 2\right)$.

Linear equation. We first assume that $\nu=0$, i.e. we solve the time-independent linear Schrödinger equation using the CNGF method. Numerical data are as follows: $\Delta t=0.1, \Delta r=8 \times 10^{-3}$. The size of the overlapping region is fixed to $\varepsilon_{\Delta r}=8 \times 10^{-2}=10 \Delta r$. According to (51), the rate of convergence for the CSWR method is expected to be approximately given by

$$
L_{\Delta r}^{C}\left(\tau_{\text {num }}\right) \approx \exp \left(-\varepsilon_{\Delta r}\left[\sqrt{2\left|\tau_{\text {num }}\right|}-V\left(R_{0}\right) \frac{1}{\sqrt{2\left|\tau_{\text {num }}\right|}}+\frac{1}{4 R_{0}^{2}} \frac{1}{\sqrt{2\left|\tau_{\text {num }}\right|}}\right]\right) .
$$

Let us remark that, for a null curvature $\kappa_{0} \rightarrow 0\left(R_{0} \rightarrow+\infty\right)$, the equation degenerates into the onedimensional Schrödinger equation, and

$$
L_{\Delta r}^{C}\left(\tau_{\text {num }}\right) \approx \exp \left(-\varepsilon_{\Delta r}\left[\sqrt{-2\left|\tau_{\text {num }}\right|}-V\left(R_{0}\right) \frac{1}{\sqrt{-2\left|\tau_{\text {num }}\right|}}\right]\right) .
$$

In Fig. 2 (left), we report the CNGF convergence time $T^{(k)}$ with respect to the Schwarz iterations for the CSWR with polar symmetry. The total number of iterations to reach the convergence of the CSWR-DDM at 
machine tolerance is equal to $k^{\mathrm{cvg}}=250$. We observe the decay of the sequence $\left(T^{(k)}\right)_{k_{0} \leqslant k \leqslant k^{\text {cvg }}}$, for $k_{0}$ large enough $\left(k_{0} \approx 200\right)$, which is in accordance with the decay assumption in Theorem 3.3. In the asymptotic regime, the $\left|\tau_{\text {num }}\right|$ belong to $\left[1 / T^{\mathrm{cvf}}, 1 / 1 \Delta t\right]$ (according to our definition of the Fourier transform). The value of $T^{(\mathrm{cvg})}$ can easily be evaluated numerically. In the numerical comparison, we then have chosen $\left|\tau_{\text {num }}\right|=1 / \Delta t$, in order to evaluate the supremum in $\left|\tau_{\text {num }}\right|$ of $L_{\Delta r}^{C}$. Fig. 2 (right) compares the numerical convergence rate obtained with the CNGF-SIE algorithm and the theoretical convergence rates (67) but written at the discrete level, i.e. we represent the $L^{2}$-norm error in time in the overlapping region. The numerical slope is given by $\approx-0.3268$, when the theoretical one, according to $(67)$ is $\approx-0.3298$.

Notice that as expected the convergence rate is numerically slowed down by the coefficient $\varepsilon_{\Delta r} \sqrt{2|\tau|} / 4 R_{0}^{2}$.
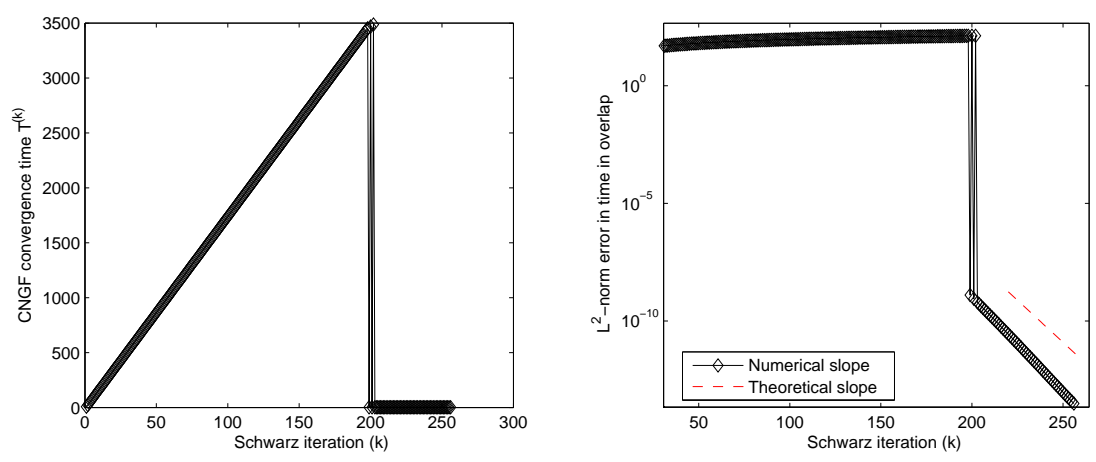

Figure 2: Two-dimensional problem with polar symmetry. Left: stopping times $T^{(k)}$ vs. CSWR iteration $k$ until convergence. Right: Comparison between the discrete versions of the estimated theoretical convergence rates (67) and numerical ones computed by the CSWR algorithm.

Nonlinear equation. In this case, $\nu \neq 0$, and the numerical data are as follows: $\Delta t=0.1, \Delta r=2.5 \times 10^{-3}$. The overlapping region has a size fixed to $\varepsilon_{\Delta r}=2.5 \times 10^{-2}=10 \Delta r$. The rate of convergence for the CSWR method is expected to be well approximated by

$$
L_{\Delta r}^{C}\left(\tau_{\text {num }}\right) \approx \exp \left(-\varepsilon_{\Delta r}\left[\sqrt{2\left|\tau_{\text {num }}\right|}-\left(V\left(R_{0}\right)+\nu\left|\phi_{s}\left(R_{0}\right)\right|^{2}\right) \frac{1}{\sqrt{2\left|\tau_{\text {num }}\right|}}+\frac{1}{4 R_{0}^{2}} \frac{1}{\sqrt{2\left|\tau_{\text {num }}\right|}}\right]\right) .
$$

In Fig. 3 (left), we report the CNGF convergence time $T^{(k)}$ vs. the Schwarz iterations for the CSWR with polar symmetry. The total number of iterations for the CSWR convergence at machine tolerance is

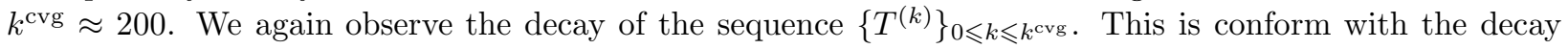
assumption made in Theorem 3.3. Fig. 3 (right) reports the numerical convergence rate obtained with the CNGF-SIE algorithm and the theoretical convergence rates (69) but written at the discrete level, i.e. we represent the $L^{2}$-norm error in time in the overlap. The numerical slope is given by $\approx-0.2919$, when the theoretical one is found to be $\approx-0.2903$, according to $(69)$.

\subsection{Numerical examples in the two-dimensional case without polar symmetry}

An exhaustive numerical illustration of the multi-dimensional theoretical results will be presented in a forthcoming paper. We propose here some preliminary results in non-symmetric two-dimensional setting. We compare the theoretical and experimental slopes of the residual error of the CSWR algorithm for computing on two-domains the ground state to the following two-dimensional nonlinear Schrödinger equation

$$
i u_{t}=-\frac{1}{2} \Delta u+V(x, y) u+\nu|u|^{2} u,
$$



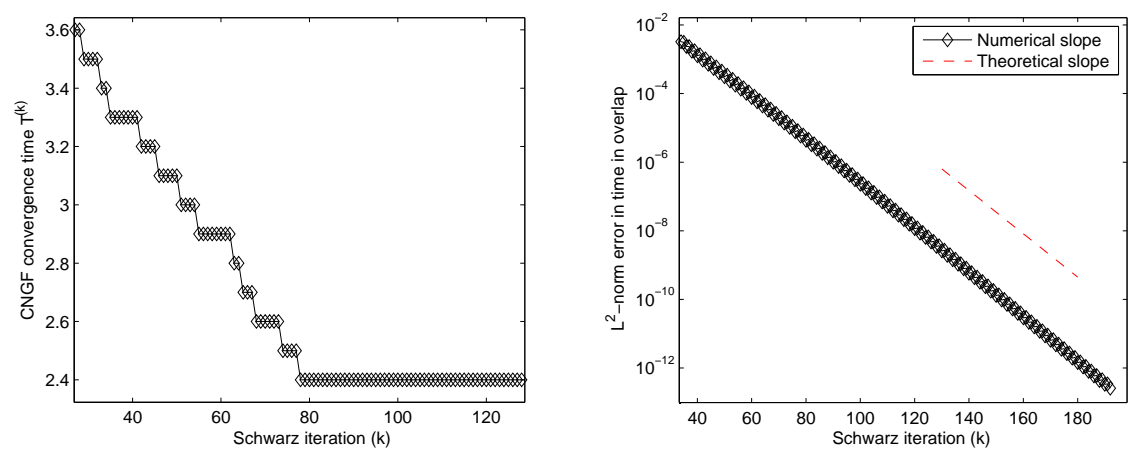

Figure 3: Two-dimensional problem with polar symmetry. Left: stopping times $T^{(k)}$ vs. CSWR iteration $k$ until convergence. Right: Comparison between the discrete versions of the estimated theoretical convergence rates (69) and numerical ones computed by the CSWR algorithm.

where $\Delta=\partial_{x}^{2}+\partial_{y}^{2}$. We take $\nu=200$ and the potentiel is the harmonic oscillator potential plus a potential of a stirrer corresponding to a far-blue detuned Gaussian laser beam [18]

$$
V(x, y)=\frac{1}{2}\left(x^{2}+y^{2}\right)+4 e^{-\left((x-1)^{2}+y^{2}\right)} .
$$

In the numerical experiment, we take the initial guess in each Schwarz iteration as

$$
\phi_{0}(x, y)=\frac{1}{\sqrt{\pi}} e^{-\left(x^{2}+y^{2}\right) / 2}
$$

The parameters of the equation and the initial guess are those of [18]. The equation is rewritten and discretized in polar coordinates $(r, \theta)$, and the global domain is the disc $\Omega_{R_{1}=6}=\{(r, \theta) \in(0,6) \times[0,2 \pi)\}$. A standard semi-implicit Euler finite difference scheme [18] is again used to approximate the equation. The total number of mesh points in the $r$-direction is $100+100-4=196$, and 60 points are used in the $\theta$ direction. Hence, the mesh step size in $r$-direction is $\Delta r=6 /(195+0.5)$ and in the $\theta$-direction $\Delta \theta=\pi / 30$. The coefficient 0.5 in the denominator of $\Delta r$ is introduced to circumvent the singularity issue at the origin. The interior and exterior domains $\Omega_{R_{0}, \varepsilon}^{ \pm}$have then 100 mesh points in the $r$-direction. The overlap region is a circular ring with 4 mesh points in the $r$-direction. Both $\Omega_{R_{0}, \varepsilon}^{+}$and $\Omega_{R_{0}, \varepsilon}^{-}$are then "cut" into 60 elementary segments in the $\theta$-direction. In the numerical test we take $\Delta t=0.025$, and set $\varepsilon_{\Delta r}=4 \Delta r=0.12$. We report in Fig. 4 (top, left) the initial guess $(k=0$ and $t=0$ ), as well as the CNGF converged solution (top, right) for $k=1$ (first Schwarz iteration). The converged solution, $k=k^{(\mathrm{cvg})}$,is reported in Fig. 4 (bottom, left) which is consistent with [18]. The residual error (66) is plotted in Fig. 4 (bottom, right) as a function of the Schwarz iteration $k$. In order to compare the numerical and theoretical rates of convergence, we fix $R_{0}$ as the radius of one of the "rings" in the center, that is $R_{0}=(100-2) \Delta r=2.99$. The theoretical convergence rate is given by (49), i.e. at the discrete level

$$
L_{\Delta r}^{C}\left(\tau_{\text {num }}\right) \approx \exp \left(-\varepsilon_{\Delta r}\left[\sqrt{2\left|\tau_{\text {num }}\right|}-\left(V\left(R_{0}\right)+\nu\left|\phi_{s}\left(R_{0}\right)\right|^{2}\right) \frac{1}{\sqrt{2\left|\tau_{\text {num }}\right|}}+\frac{1}{4 R_{0}^{2}} \frac{1}{\sqrt{2\left|\tau_{\text {num }}\right|}}\right]\right) .
$$

The lowest values of $V$ and $\left|\phi_{s}\right|^{2}$ on the overlapping ring are respectively $V\left(R_{0}\right)=4.49$ and $\left|\phi_{s}\left(R_{0}\right)\right|^{2}=0.017$. To sum it all up and by taking $\tau_{\text {num }}=-1 / \Delta t$, we have $\log \left(L_{\Delta r}^{C}\left(\tau_{\text {num }}\right)\right) \approx-0.98$, which is very close to the estimated numerical slope $\approx-1.03$.

\section{Concluding remarks}

In this paper, we have analyzed an asymptotic convergence of the CSWR method for solving the timeindependent LSE/GPE by using the CNGF method. Through approximations and by using techniques 

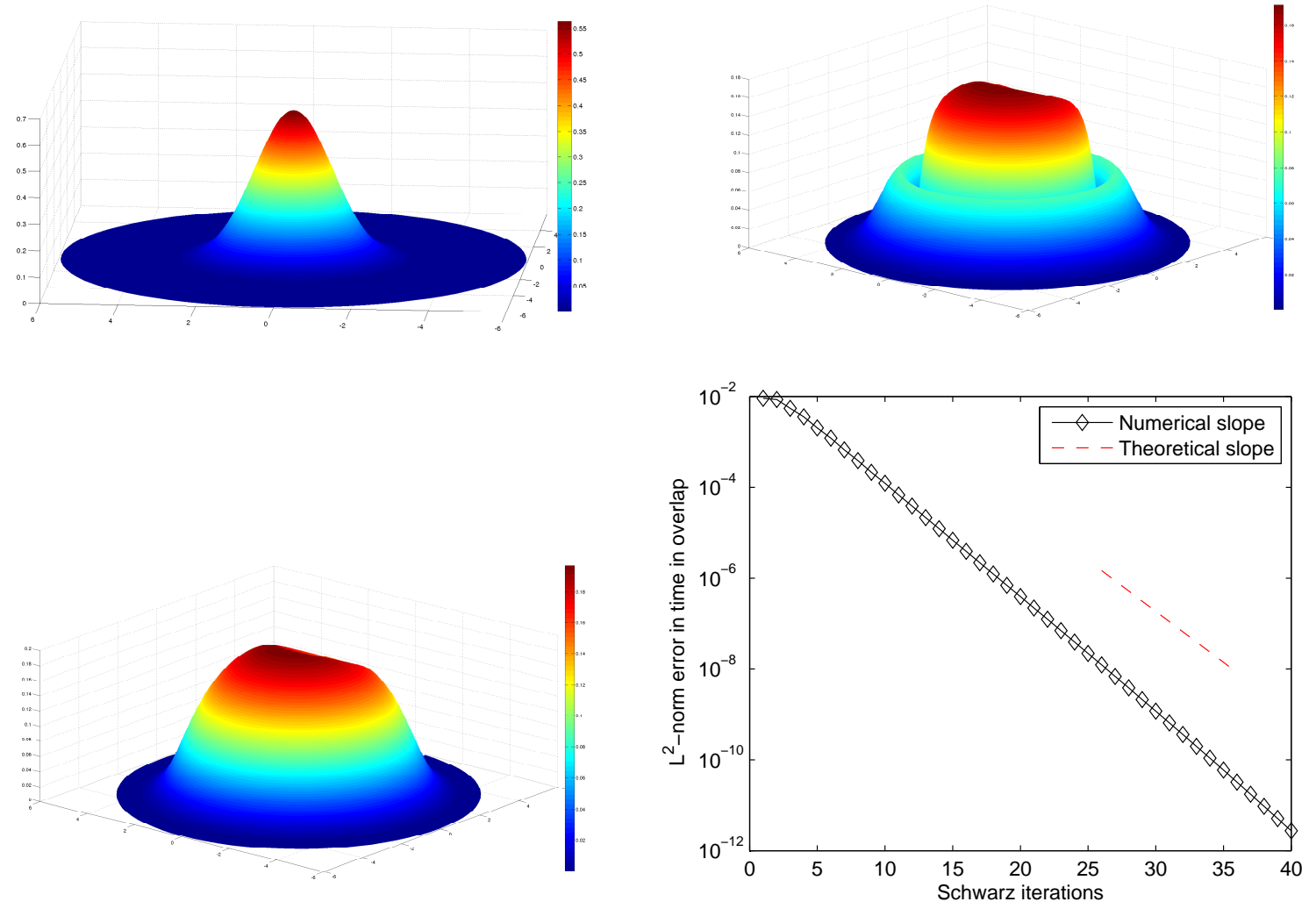

Figure 4: Two-dimensional problem without polar symmetry. (Top, left) Initial guess $(k=0, t=0)$, $\phi_{0}$. (Top, right) CNGF converged solution for $k=1, \phi^{n^{\mathrm{cvg}},(1)}$. (Bottom, left) Converged reconstructed solution $\phi^{\mathrm{cvg}}$. (Bottom, right) Comparison between the discrete versions of the estimated theoretical residual error (67), and numerical ones computed by the CSWR algorithm in a 2-d non-symmetric configuration.

from pseudodifferential calculus, we have derived some accurate convergence rates for the CSWR-DDM. Extending the one-dimensional analysis from [12], we have exhibited in particular the effect of the curvature of the subdomain boundary on the SWR convergence rates. Some preliminary two-dimensional simulations with and without polar-symmetry have validated these analytical results. Let us remark that the approach provided in this paper can also be applied to the LSE/GPE in real-time, by replacing $\tau$ (respectively $t$ ) by $-i \tau$ (respectively $-i t$ ) in all the derived formulae and skipping the normalization step. The latter would naturally require a finer analysis in the $E$-quasi elliptic, hyperbolic and glancing regions. We also notice that the technical tools and overall strategy can be extended to other kinds of wave equations and to higher dimensional problems.

In a forthcoming paper, some exhaustive numerical simulations and analysis will be presented to i) validate the analysis developed here in more complex numerical configurations, and ii) to provide stable and accurate numerical LSE/GPE solvers that use SWR-DDM in the real- and imaginary-time settings.

\section{APPENDIX: Proof. of Proposition 3.5}

We first recall the fundamental symbolic equation.

$$
\begin{aligned}
i \partial_{r} \lambda^{+}+i \kappa \lambda^{+} & +\sum_{|\alpha|=0}^{+\infty} \frac{(-i)^{|\alpha|}}{\alpha !} \partial_{(\xi, \tau)}^{\alpha} \lambda^{+} \partial_{(t, r)}^{\alpha} \lambda^{+} \\
& =-i \tau-h^{-2} \xi^{2}+i h^{-1}\left(\partial_{s} h^{-1}\right) \xi-V_{r}
\end{aligned}
$$


By identifying the zeroth-order symbols

$$
\begin{aligned}
& i \partial_{r} \lambda_{0}^{+}+i \kappa \lambda_{0}^{+}+2 \lambda_{1}^{+} \lambda_{-1}^{+} \\
&-i \partial_{(\xi, \tau)} \lambda_{0}^{+} \partial_{(t, s)} \lambda_{1}^{+}-i \partial_{(\xi, \tau)} \lambda_{1}^{+} \partial_{(t, s)} \lambda_{0}^{+}-\partial_{(\xi, \tau)}^{2} \lambda_{1}^{+} \partial_{(t, s)}^{2} \lambda_{1}^{+} / 2=-V_{r},
\end{aligned}
$$

one gets

$$
=\frac{-V_{r}-i \partial_{r} \lambda_{0}^{+}-i \kappa \lambda_{0}^{+}+i \partial_{(\xi, \tau)} \lambda_{0}^{+} \partial_{(t, s)} \lambda_{1}^{+}+i \partial_{(\xi, \tau)} \lambda_{1}^{+} \partial_{(t, s)} \lambda_{0}^{+}+\partial_{(\xi, \tau)}^{2} \lambda_{1}^{+} \partial_{(t, s)}^{2} \lambda_{1}^{+} / 2}{2 \lambda_{1}^{+}}
$$

As in the case $\varepsilon=0$, we intend to truncate the symbols expression at order -1 in $\tau$. Then, from the expression of $\lambda_{0}^{+}$, we will get an approximation of $\lambda_{-1}^{+}$. Since $1 / \lambda_{1}^{+}$is of order $-1 / 2$ in $\tau$, we have to determine the contribution of order $-1 / 2$ appearing in the numerator of the above equation to estimate $\left(\lambda_{-1}^{+}\right)_{(-1)}$ (of order -1 in $\tau$ ). In this goal, we first determine from $(24)$ :

$$
\begin{aligned}
\partial_{s} \lambda_{0}^{+}=-\frac{i \partial_{s} \kappa}{2}+ & \frac{i}{4} \frac{\xi^{2} \partial_{s r}^{2} h^{-2}\left(-i \tau-h^{-2} \xi^{2}+i h^{-1}\left(\partial_{s} h^{-1}\right) \xi-V_{r}\right)}{\left(-i \tau-h^{-2} \xi^{2}+i h^{-1}\left(\partial_{s} h^{-1}\right) \xi-V_{r}\right)^{2}} \\
& -\frac{i}{4} \frac{\left(\xi \partial_{s} h^{-2}+i \partial_{s}\left(h^{-1}\left(\partial_{s} h^{-1}\right)\right)\right) \xi^{3} \partial_{r} h^{-2}}{\left(-i \tau-h^{-2} \xi^{2}+i h^{-1}\left(\partial_{s} h^{-1}\right) \xi-V_{r}\right)^{2}} \\
& -\frac{i}{8} \frac{2 \xi^{3} \partial_{s}\left(h^{-2}\left(\partial_{s} h^{-2}\right)\right)\left(-i \tau-h^{-2} \xi^{2}+i h^{-1}\left(\partial_{s} h^{-1}\right) \xi-V_{r}\right)}{\sqrt{-i \tau-h^{-2} \xi^{2}+i h^{-1}\left(\partial_{s} h^{-1}\right) \xi-V_{r}}} \\
& +\frac{i}{8} \frac{3 h^{-2} \xi^{4}\left(\partial_{s} h^{-2}\right)\left(i \partial_{s}\left(h^{-1}\left(\partial_{s} h^{-1}\right)\right)-\xi \partial_{s} h^{-2}\right)}{\sqrt{-i \tau-h^{-2} \xi^{2}+i h^{-1}\left(\partial_{s} h^{-1}\right) \xi-V_{r}^{5}}}
\end{aligned}
$$

and

$$
\begin{aligned}
\partial_{\xi} \lambda_{0}^{+}=\frac{i 2 \xi(}{4} \frac{\left(\partial_{r} h^{-2}\right)\left(-i \tau-h^{-2} \xi^{2}+i h^{-1}\left(\partial_{s} h^{-1}\right) \xi-V_{r}\right)}{\left(-i \tau-h^{-2} \xi^{2}+i h^{-1}\left(\partial_{s} h^{-1}\right) \xi-V_{r}\right)^{2}} \\
-\frac{i\left(\partial_{r} h^{-2}\right) \xi^{2}\left(-2 h^{-2} \xi+i h^{-1}\left(\partial_{s} h^{-1}\right)\right)}{4\left(-i \tau-h^{-2} \xi^{2}+i h^{-1}\left(\partial_{s} h^{-1}\right) \xi-V_{r}\right)^{2}} \\
-\frac{3 i h^{-2}\left(\partial_{s} h^{-2}\right) \xi^{2}\left(-i \tau-h^{-2} \xi^{2}+i h^{-1}\left(\partial_{s} h^{-1}\right) \xi-V_{r}\right)}{4} \\
+\frac{3 i h^{-2} \xi^{3}\left(\partial_{s} h^{-2}\right)\left(i h^{-1}\left(\partial_{s} h^{-1}\right)-2 h^{-2} \xi\right)}{8} \sqrt{-i \tau-h^{-2} \xi^{2}+i h^{-1}\left(\partial_{s} h^{-1}\right) \xi-V_{r}}
\end{aligned}
$$

Now for any $j \in \mathbb{N}^{*}$, we have: $\partial_{t}^{j} \lambda_{1}^{+}=\partial_{t}^{j} \lambda_{0}^{+}=0$. Moreover, some direct computations lead to

$$
\partial_{s} \lambda_{1}^{+}=\frac{\partial_{s} h^{-2} \xi^{2}+\partial_{s} V_{r}}{2 \sqrt{-i \tau-h^{-2} \xi^{2}-V_{r}}}, \quad \partial_{\xi} \lambda_{1}^{+}=\frac{h^{-2} \xi}{\sqrt{-i \tau-h^{-2} \xi^{2}-V_{r}}} .
$$

We deduce that the following equalities hold

$$
\left(\partial_{s} \lambda_{0}^{+}\right)_{(0)}=-\frac{i \partial_{s} \kappa}{2}, \quad\left(\partial_{\xi} \lambda_{0}^{+}\right)_{(0)}=0, \quad\left(\partial_{s} \lambda_{0}^{+}\right)_{(-1 / 2)}=0, \quad\left(\partial_{\xi} \lambda_{0}^{+}\right)_{(-1 / 2)}=0 .
$$

In addition, we have

$$
\partial_{\xi}^{2} \lambda_{1}^{+}=\frac{h^{-2}\left(-i \tau-h^{-2} \xi^{2}-V_{r}\right)+h^{-4} \xi^{2}}{\sqrt{-i \tau-h^{-2} \xi^{2}-V_{r}}}
$$


and

$$
\partial_{s}^{2} \lambda_{1}^{+}=\frac{2\left(\xi^{2} \partial_{s}^{-2} h^{-2}+\partial_{s}^{2} V_{r}\right)\left(-i \tau-h^{-2} \xi^{2}-V_{r}\right)+\left(\xi^{2} \partial_{s} h^{-2}\right)^{2}}{4{\sqrt{-i \tau-h^{-2} \xi^{2}-V_{r}}}^{3}} .
$$

Then, we conclude that: $\left(\partial_{\xi}^{2} \lambda_{1}^{+} \partial_{s}^{2} \lambda_{1}^{+}\right)_{(0)}=\left(\partial_{\xi}^{2} \lambda_{1}^{+} \partial_{s}^{2} \lambda_{1}^{+}\right)_{(-1 / 2)}=0$. We deduce that for $\tau \in \mathbb{R}_{\infty}$

$$
\left(\partial_{s} \lambda_{0}^{+} \partial_{\xi} \lambda_{1}^{+}\right)_{(-1 / 2)}=-i \partial_{s} \kappa \frac{h^{-2} \xi}{2 \sqrt{-i \tau}}
$$

and we finally have for large $\tau \in \mathbb{R}_{\infty}$

$$
\left(\widetilde{\lambda}_{-1}^{+}\right)_{(-1)}=-e^{-\pi / 4} \frac{\partial_{r} \kappa}{4 \sqrt{-\tau}}-e^{-\pi / 4} \frac{\kappa^{2}}{4 \sqrt{-\tau}}-i \partial_{s} \kappa \frac{h^{-2} \xi}{2 \tau} .
$$

In order to evaluate $\left(\lambda_{-2}^{+}\right)_{(-1)}$, we again use the fundamental relation (73), equaling the symbols of order $-1$

$$
\lambda_{-2}^{+}=\frac{-i \partial_{r} \lambda_{-1}^{+}-i \kappa \lambda_{-1}^{+}+i \partial_{(\xi, \tau)} \lambda_{0}^{+} \partial_{(t, s)} \lambda_{0}^{+}+i \partial_{(\xi, \tau)} \lambda_{1}^{+} \partial_{(t, s)} \lambda_{-1}^{+}+\partial_{(\xi, \tau)}^{2} \lambda_{1}^{+} \partial_{(t, s)}^{2} \lambda_{0}^{+} / 2}{2 \sqrt{-i \tau-h^{-2} \xi^{2}-V_{r}}}
$$

As order of $1 / \lambda_{1}^{+}$is of order $-1 / 2$ in $\tau$, in order to determine $\left(\lambda_{-2}^{+}\right)_{-1}$, we also need to estimate the order $-1 / 2$ contribution of the numerator in the expression above. We skip the details and directly get

$$
\left(\partial_{r} \lambda_{1}^{+}\right)_{(-1 / 2)}=\frac{\partial_{r} h^{-2} \xi^{2}+\partial_{r} V_{r}}{2 \sqrt{-i \tau-h^{-2} \xi^{2}-V_{r}}}
$$

and, for $\tau \in \mathbb{R}_{\infty}$,

$$
\left(\partial_{s}^{2} \lambda_{0}^{+} \partial_{\xi}^{2} \lambda_{1}^{+}\right)_{(-1 / 2)}=-\frac{i}{2 \sqrt{-\tau}} \partial_{s}^{2} \kappa .
$$

Following the same strategy as above, we obtain

$$
\left(\widetilde{\lambda}_{-2}^{+}\right)_{(-1)}=-\frac{1}{4} \frac{\partial_{\mathbf{n}} V_{r}}{\tau}-\frac{1}{8} \frac{\partial_{s}^{2} \kappa}{\tau}-\frac{1}{8} \frac{\kappa^{3}}{\tau} .
$$

This concludes the proof of the first part of the proposition.

We then have to evaluate $\kappa(0, s), \partial_{r} \kappa(0, s), \partial_{s} \kappa(0, s), \partial_{s}^{2} \kappa(0, s)$. At $\Gamma_{\varepsilon, r}^{ \pm}$we have

$$
\kappa(r, s)= \pm\left(1 \pm(\varepsilon / 2-r) \kappa_{0}(s)\right)^{-1} \kappa_{0}(s),
$$

then, at $\Gamma_{\varepsilon}^{ \pm}: \kappa(0, s)= \pm\left(1 \pm \varepsilon / 2 \kappa_{0}(s)\right)^{-1} \kappa_{0}(s)$. Next, we write that

$$
\partial_{r} \kappa(r, s)=\frac{\kappa_{0}^{2}(s)}{1 \pm(\varepsilon / 2-r) \kappa_{0}(s)}
$$

which provides

$$
\partial_{r} \kappa(0, s)=\frac{\kappa_{0}^{2}(s)}{1 \pm \varepsilon / 2 \kappa_{0}(s)} .
$$

Similarly, one gets

$$
\partial_{s} \kappa(r, s)= \pm \frac{\partial_{s} \kappa_{0}(s)}{\left(1 \pm(\varepsilon / 2-r) \kappa_{0}(s)\right)^{2}}
$$


leading to

$$
\partial_{s} \kappa(0, s)= \pm \frac{\partial_{s} \kappa_{0}(s)}{\left(1 \pm \varepsilon / 2 \kappa_{0}(s)\right)^{2}}
$$

Finally, some calculations show that

$$
\partial_{s}^{2} \kappa(r, s)= \pm \frac{\partial_{s}^{2} \kappa_{0}(s) \pm(\varepsilon / 2-r)\left(\kappa_{0}(s) \partial_{s}^{2} \kappa_{0}(s)-\left(\partial_{s} \kappa_{0}(s)\right)^{2}\right)}{\left(1 \pm(\varepsilon / 2-r) \kappa_{0}(s)\right)^{3}}
$$

and

$$
\partial_{s}^{2} \kappa(0, s)= \pm \frac{2 \partial_{s}^{2} \kappa_{0}(s) \pm \varepsilon\left(\kappa_{0}(s) \partial_{s}^{2} \kappa_{0}(s)-\left(\partial_{s} \kappa_{0}(s)\right)^{2}\right)}{2\left(1 \pm \varepsilon \kappa_{0}(s) / 2\right)^{3}} .
$$

This concludes the proof.

\section{References}

[1] M. Al-Khaleel, A.E. Ruehli, and M.J. Gander, Optimized waveform relaxation methods for longitudinal partitioning of transmission lines, IEEE Transactions on Circuits and Systems 56 (2009), 1732-1743.

[2] X. Antoine, W. Bao, and C. Besse, Computational methods for the dynamics of the nonlinear Schrödinger/Gross-Pitaevskii equations, Comput. Phys. Comm. 184 (2013), no. 12, 2621-2633.

[3] X. Antoine and C. Besse, Construction, structure and asymptotic approximations of a microdifferential transparent boundary condition for the linear Schrödinger equation, J. Math. Pures Appl. (9) 80 (2001), no. 7, 701-738. MR 1846022 (2003h:35213)

[4] X. Antoine, C. Besse, and S. Descombes, Artificial boundary conditions for one-dimensional cubic nonlinear Schrödinger equations, SIAM J. Numer. Anal. 43 (2006), no. 6, 2272-2293 (electronic). MR 2206436 (2006i:35336)

[5] X. Antoine, C. Besse, and P. Klein, Absorbing boundary conditions for the one-dimensional Schrödinger equation with an exterior repulsive potential, J. Comput. Phys. 228 (2009), no. 2, 312-335. MR 2479925 (2009j:65177)

[6] _ Absorbing boundary conditions for the two-dimensional Schrödinger equation with an exterior potential. Part I: Construction and a priori estimates, Math. Models Methods Appl. Sci. 22 (2012), no. 10, 1250026, 38. MR 2974164

[7] X. Antoine, C. Besse, and V. Mouysset, Numerical schemes for the simulation of the two-dimensional Schrödinger equation using non-reflecting boundary conditions, Math. Comp. 73 (2004), no. 248, 17791799 (electronic). MR 2059736 (2005c:65067)

[8] X. Antoine, C. Besse, and J. Szeftel, Towards accurate artificial boundary conditions for nonlinear PDEs through examples, Cubo 11 (2009), no. 4, 29-48. MR 2571793 (2010j:35002)

[9] X. Antoine and R. Duboscq, GPELab, a Matlab toolbox to solve Gross-Pitaevskii equations I: Computation of stationary solutions, Computer Physics Communications 185 (2014), no. 11, 2969-2991.

[10] _ Robust and efficient preconditioned Krylov spectral solvers for computing the ground states of fast rotating and strongly interacting Bose-Einstein condensates, J. of Comput. Phys. 258C (2014), 509-523. 
[11] _ Modeling and computation of Bose-Einstein condensates: stationary states, nucleation, dynamics, stochasticity, in Nonlinear Optical and Atomic Systems: at the Interface of Mathematics and Physics, CEMPI Subseries, 1st Volume, 2146, Lecture Notes in Mathematics, Springer, 2015, pp. 49-145.

[12] X. Antoine and E. Lorin, An analysis of schwarz waveform relaxation domain decomposition methods for the imaginary-time linear Schrödinger and Gross-Pitaevskii equations, Submitted (2015).

[13] _ Lagrange Schwarz waveform relaxation domain decomposition methods for linear and nonlinear quantum wave problems, Applied Math. Lett. 57 (2016), 38-45.

[14] X. Antoine, E. Lorin, and A.D. Bandrauk, Domain decomposition method and high-order absorbing boundary conditions for the numerical simulation of the time dependent schrödinger equation with ionization and recombination by intense electric field, Journal of Scientific Computing 64 (2015), no. 3, 620-646.

[15] W. Bao, Ground states and dynamics of multicomponent Bose-Einstein condensates, Multiscale Modeling \& Simulation 2 (2004), no. 2, 210-236.

[16] W. Bao and Y. Cai, Mathematical theory and numerical methods for Bose-Einstein condensation, Kinetic and Related Models 6 (2013), no. 1, 1-135.

[17] W. Bao, I-L. Chern, and F.Y. Lim, Efficient and spectrally accurate numerical methods for computing ground and first excited states in Bose-Einstein condensates, J. of Comput. Phys. 219 (2006), no. 2, $836-854$.

[18] W. Bao and Q. Du, Computing the ground state solution of Bose-Einstein condensates by a normalized gradient flow, SIAM J. Sci. Comput. 25 (2004), no. 5, 1674-1697. MR 2087331 (2005f:82117)

[19] W. Bao and W. Tang, Ground-state solution of Bose-Einstein condensate by directly minimizing the energy functional, J. Comput. Phys. 187 (2003), no. 1, 230-254. MR 1977785 (2004g:82064)

[20] D. Baye and J-M. Sparenberg, Resolution of the Gross-Pitaevskii equation with the imaginary-time method on a Lagrange mesh, Physical Review E 82 (2010), no. 5, 056701.

[21] C. Besse and F. Xing, Domain decomposition algorithms for two dimensional linear Schrödinger equation, submitted (2015).

[22] _ Schwarz waveform relaxation method for one dimensional Schrödinger equation with general potential, submitted (2015).

[23] M. L. Chiofalo, S. Succi, and M. P. Tosi, Ground state of trapped interacting Bose-Einstein condensates by an explicit imaginary-time algorithm, Physical Review E 62 (2000), no. 5, 7438.

[24] V. Dolean, P. Jolivet, and F. Nataf, An introduction to domain decomposition methods: theory and parallel implementation, 2015.

[25] M. Gander and L. Halpern, Optimized Schwarz waveform relaxation methods for advection reaction diffusion problems, SIAM J. Num. Anal. 45 (2007), no. 2.

[26] M.J. Gander, Overlapping Schwarz for linear and nonlinear parabolic problems, Proceedings of the 9th International Conference on Domain decomposition, 1996, pp. 97-104.

[27] — Optimal Schwarz waveform relaxation methods for the one-dimensional wave equation, SIAM J. Numer. Anal. 41 (2003), 1643-1681.

[28] _ Optimized Schwarz methods, SIAM J. Numer. Anal. 44 (2006), 699-731. 
[29] _ Optimized Schwarz waveform relaxation methods for advection diffusion problems, SIAM J. Numer. Anal. (2007), 666-697.

[30] M.J. Gander, L. Halpern, and F. Nataf, Optimal convergence for overlapping and non-overlapping Schwarz waveform relaxation, DDM.org, Augsburg, 1999, pp. 27-36.

[31] M.J. Gander, F. Kwok, and B. Mandal, Dirichlet-Neumann and Neumann-Neumann waveform relaxation algorithms for parabolic problems, submitted (2015).

[32] L. Halpern and J. Szeftel, Optimized and quasi-optimal Schwarz waveform relaxation for the onedimensional Schrödinger equation, Math. Models Methods Appl. Sci. 20 (2010), no. 12, 2167-2199. MR 2755497 (2012d:35307)

[33] R. Lascar, Propagation des singularités des solutions d'équations pseudo-différentielles quasi homogènes, Ann. Inst. Fourier (Grenoble) 27 (1977), no. 2, vii-viii, 79-123. MR 0461592 (57 \#1577)

[34] J.-L. Lions and E. Magenes, Non-homogeneous boundary value problems and applications. Vol. II, Springer-Verlag, New York-Heidelberg, 1972, Translated from the French by P. Kenneth, Die Grundlehren der mathematischen Wissenschaften, Band 182. MR 0350178

[35] E. Lorin, X. Yang, and X. Antoine, Frozen gaussian approximation based domain decomposition methods for the linear Schrödinger equation beyond the semi-classical regime, J. Comput. Phys. 315 (2016), 221237.

[36] B. Mandal, A time-dependent Dirichlet-Neumann method for the heat equation, p. 2014.

[37] L. Nirenberg, Lectures on linear partial differential equations, American Mathematical Society, Providence, R.I., 1973. MR 0450755 (56 \#9048)

[38] C. J. Pethick and H. Smith, Bose-Einstein condensation in dilute gases, Cambridge University Press, 2002.

[39] L. P. Pitaevskii and S. Stringari, Bose-Einstein condensation, vol. 116, Clarendon press, 2003.

[40] R. Zeng and Y. Zhang, Efficiently computing vortex lattices in rapid rotating Bose-Einstein condensates, Computer Physics Communications 180 (2009), no. 6, 854-860. 